October 29, 2018

UMDEPP 12-003

\title{
The Real Anatomy of Complex Linear Superfields
}

\author{
S.J. Gates, Jr.*, J. Hallett**, T.Hübsch ${ }^{\dagger \dagger}$ and K.Stiffler* \\ * Center for String and Particle Theory, Dept. of Physics, \\ University of Maryland, College Park, MD 20472, \\ ** Department of Mathematics, Williams College, Williamstown, MA 01267 USA \\ $\ddagger$ Dept. of Physics, University of Central Florida, Orlando, FL \\ ${ }^{\dagger}$ Dept. of Physics $\&$ Astronomy, Howard University, Washington, DC 20059
}

\begin{abstract}
Recent work on classification of off-shell representations of $N$-extended worldline supersymmetry without central charges has uncovered an unexpectedly vast number-trillions of even just (chromo)topology types - of so called adinkraic supermultiplets. Herein, we show by explicit analysis that a long-known but rarely used representation, the complex linear supermultiplet, is not adinkraic, cannot be decomposed locally, but may be reduced by means of a Wess-Zumino type gauge. This then indicates that the already unexpectedly vast number of adinkraic off-shell supersymmetry representations is but the proverbial tip of the iceberg.
\end{abstract}

\section{Introduction, Results and Summary}

\subsection{History and Organization}

Refs. $[1,2,3,4,5,6]$ developed a detailed classification of a huge class $\left(\sim 10^{12}\right.$ for no more than 32 supersymmetries) of adinkraic off-shell worldline supermultiplets, wherein each supercharge maps each component field to precisely one other component field or its $\tau$-derivative. These supermultiplets are faithfully depicted by graphs called Adinkras; see also Refs. [7,8,9,10,11,12,13]. That a basis of supercharges and a compatible basis of component fields that exhibits such an acute syzygy can even exist is by no means guaranteed. Herein, we demonstrate that an oft-ignored "red-headed stepchild" in the family of famous and familiar supermultiplets, the complex linear supermultiplet [14] ${ }^{1}-C L S$, for short, in fact is not adinkraic. This, in fact, had to be the case, since the $12+12$ component fields of CLS cannot possibly span a hypercubical chromotopology, as required by Theorem 4.1 of Ref. [4] .

In the remainder of this introduction, we specify the notation and conventions. Section 2 analyzes the real scalar supermultiplet $(R S S)$ and real pseudoscalar supermultiplet $(R P S)$ in their real/Majorana structure in 3+1-dimensional spacetime and reduces them to their worldlines, i.e., 0brane. Section 3 merges the RSS and RPS into the CLS and analyzes the resulting 3+1-dimensional spacetime structure and 0-brane reduction, following a similar pattern to Section 2. Section 4 presents the supersymmetric action for the RSS, RPS, and CLS and concludes with a discussion of the implications of this result.

\footnotetext{
${ }^{1}$ This was also called the non-minimal chiral supermultiplet in Ref. [15].
} 


\subsection{Definitions}

Complex linear superfields are defined by the property that they are annihilated by a Lorentzinvariant "square" of the conjugate superderivatives. We focus on the most familiar case, of simple supersymmetry in $1+3$-dimensional spacetime that has a total of $N=4$ supercharges. We consider the worldline (0-brane) dimensional reduction of this particle, and so operate with the (1|4)-supersymmetry algebra

$$
\left.\begin{array}{rlrl}
\left\{Q_{I}, Q_{J}\right\} & =2 \delta_{I J} H, & {\left[H, Q_{I}\right]} & =0, \\
Q_{I}^{\dagger} & =Q_{I}, & H^{\dagger} & =H,
\end{array}\right\} \quad I, J=1, \cdots, 4,
$$

were $H=i \hbar \partial_{\tau}$. We also have the superderivatives:

$$
\left.\begin{array}{rl}
\left\{\mathrm{D}_{I}, \mathrm{D}_{J}\right\} & =2 \delta_{I J} H, \quad\left[H, \mathrm{D}_{I}\right]=0, \\
\mathrm{D}_{I}^{\dagger} & =-\mathrm{D}_{I}, \quad\left\{Q_{I}, \mathrm{D}_{J}\right\}=0,
\end{array}\right\} \quad I, J=1, \cdots, 4 .
$$

Instead of this real basis, we may also use the complex (and nilpotent) basis of Hermitian pairs:

$$
\left.\begin{array}{l}
\boldsymbol{Q}_{\alpha}:=\frac{1}{2}\left(Q_{\alpha}+i Q_{2+\alpha}\right), \quad \overline{\boldsymbol{Q}}_{\dot{\alpha}}:=\boldsymbol{Q}_{\alpha}^{\dagger}=\frac{1}{2}\left(Q_{\dot{\alpha}}-i Q_{2+\dot{\alpha}}\right), \\
\boldsymbol{D}_{\alpha}:=\frac{1}{2}\left(\mathrm{D}_{\alpha}+i \mathrm{D}_{2+\alpha}\right), \quad \overline{\boldsymbol{D}}_{\dot{\alpha}}:=\boldsymbol{D}_{\alpha}^{\dagger}=\frac{1}{2}\left(\mathrm{D}_{\dot{\alpha}}-i \mathrm{D}_{2+\dot{\alpha}}\right),
\end{array}\right\} \quad \alpha, \dot{\alpha}=1,2 .
$$

With this basis, the algebra (1.1) and (1.2) becomes:

$$
\left\{\boldsymbol{Q}_{\alpha}, \overline{\boldsymbol{Q}}_{\dot{\beta}}\right\}=\delta_{\alpha \dot{\beta}} H, \quad\left\{\boldsymbol{D}_{\alpha}, \overline{\boldsymbol{D}}_{\dot{\beta}}\right\}=\delta_{\alpha \dot{\beta}} H,
$$

with all other (anti)commutators vanishing. In particular, note that

$$
\boldsymbol{Q}_{\alpha}^{2}=0=\overline{\boldsymbol{Q}}_{\dot{\beta}}^{2}, \text { and } \boldsymbol{D}_{\alpha}^{2}=0=\overline{\boldsymbol{D}}_{\dot{\beta}}^{2}
$$

We will also need that

$$
Q_{I}=i \mathrm{D}_{I}-2 i \delta_{I J} \theta^{J} H, \quad \text { and } \quad \mathrm{D}_{I}=-i Q_{I}-2 \delta_{I J} \theta^{J} H
$$

where $\theta^{I}$ provide the fermionic extension to (space)time into superspace. When applied on superfields (general functions over superspace), the $\mathrm{D}_{I}$ act as left-derivatives while the $Q_{I}$ act as right-derivatives. Owing to this and with the sign conventions and definitions from Ref. [16], we have that

$$
Q_{I} \phi=i \mathrm{D}_{I} \boldsymbol{\Phi} \mid, \quad \text { and } \quad Q_{I} \psi=-i \mathrm{D}_{I} \boldsymbol{\Psi} \mid,
$$

where $\boldsymbol{\Phi} \mid=\phi(\boldsymbol{\Psi} \mid=\psi)$ is an arbitrary bosonic (fermionic) functional-differential expression, and $\boldsymbol{\Phi}$ $(\boldsymbol{\Psi})$ the appropriate superfield expression defining $\phi(\psi)$ by means of the superspace $\rightarrow$ spacetime projection denoted by the "|" right-delimiter. Owing to the relations (1.7), a supermultiplet may be represented interchangeably:

$$
\begin{aligned}
& \text { as }\left\{\left(\boldsymbol{\Phi}\left|\boldsymbol{\Psi}_{I}\right| \cdots\right): \quad \mathrm{D}_{I} \boldsymbol{\Phi}=i \boldsymbol{\Psi}_{I}, \quad \mathrm{D}_{I} \boldsymbol{\Psi}_{J}=\delta_{I J} \dot{\boldsymbol{\Phi}}+\ldots, \quad \text { etc. }\right\} \text {, } \\
& \text { as }\left\{\left(\phi\left|\psi_{I}\right| \cdots\right): Q_{I}(\phi)=-\psi_{I}, Q_{I}\left(\psi_{J}\right)=-i \delta_{I J} \dot{\phi}+\ldots, \quad \text { etc. }\right\} \text {, } \\
& \text { or as }\left\{\left(\phi\left|\psi_{I}\right| \cdots\right): \delta_{e}(\phi)=i \epsilon^{I} \psi_{I}, \quad \delta_{e}\left(\psi_{J}\right)=-\epsilon^{I} \delta_{I J} \dot{\phi}+\ldots, \text { etc. }\right\} \text {. }
\end{aligned}
$$


Relying on the (1.8a)-(1.8c) correspondence, we may also specify supermultiplets in the latter representation, (1.8a), as a manifestly supersymmetric (recall: $\left\{Q_{I}, \mathrm{D}_{J}\right\}=0$ ) closed system of superdifferential relations between component superfields, the lowest component of each of which is the corresponding (ordinary spacetime) component field.

We will therefore write our transformation laws in component notation in terms of the superderivative $\mathrm{D}_{I}$. On way to our various 0 -brane reductions, we will always start with $4 D, \mathcal{N}=1$ transformation laws written in terms of $\mathrm{D}_{a}$ with $a=1,2,3,4$ a Majorana fermionic index. Throughout the paper, we denote the number of supersymmetries on the 0-brane as $N=4 \mathcal{N}$, where $\mathcal{N}$ is always the number of supersymmetries in $4 D$. We will use the real representation of the $\gamma$ matrices as in Refs. [17,18]

$$
\begin{aligned}
\left(\gamma^{0}\right)_{a}^{b}= & \left(\begin{array}{cccc}
0 & 1 & 0 & 0 \\
-1 & 0 & 0 & 0 \\
0 & 0 & 0 & -1 \\
0 & 0 & 1 & 0
\end{array}\right), \quad\left(\gamma^{1}\right)_{a}^{b}=\left(\begin{array}{cccc}
0 & 1 & 0 & 0 \\
1 & 0 & 0 & 0 \\
0 & 0 & 0 & 1 \\
0 & 0 & 1 & 0
\end{array}\right) \\
\left(\gamma^{2}\right)_{a}^{b} & =\left(\begin{array}{cccc}
0 & 0 & 0 & -1 \\
0 & 0 & 1 & 0 \\
0 & 1 & 0 & 0 \\
-1 & 0 & 0 & 0
\end{array}\right), \quad\left(\gamma^{3}\right)_{a}^{b}=\left(\begin{array}{cccc}
1 & 0 & 0 & 0 \\
0 & -1 & 0 & 0 \\
0 & 0 & 1 & 0 \\
0 & 0 & 0 & -1
\end{array}\right) \\
\left(\gamma^{5}\right)_{a}^{b} \equiv & =i\left(\gamma^{0} \gamma^{1} \gamma^{2} \gamma^{3}\right)_{a}^{b}=\left(\begin{array}{cccc}
0 & 0 & 0 & i \\
0 & 0 & -i & 0 \\
0 & i & 0 & 0 \\
-i & 0 & 0 & 0
\end{array}\right)
\end{aligned}
$$

where the fermionic indices are raised and lowered by the spinor metric $C_{a b}$ and inverse spinor metric $C^{a b}$

$$
C_{a b}=\left(\begin{array}{cccc}
0 & -1 & 0 & 0 \\
1 & 0 & 0 & 0 \\
0 & 0 & 0 & 1 \\
0 & 0 & -1 & 0
\end{array}\right) \quad, \quad C^{a b}=\left(\begin{array}{cccc}
0 & -1 & 0 & 0 \\
1 & 0 & 0 & 0 \\
0 & 0 & 0 & 1 \\
0 & 0 & -1 & 0
\end{array}\right)
$$

according to the convention

$$
\boldsymbol{\zeta}_{a}=\boldsymbol{\zeta}^{b} C_{b a} \quad, \quad \boldsymbol{\zeta}^{a}=C^{a b} \boldsymbol{\zeta}_{b}
$$

which satisfy

$$
C_{a c} C^{b c}=\delta_{a}^{b}
$$

\section{The Real Scalar and Pseudoscalar Supermultiplets}

In this section we will introduce both the real scalar and real pseudoscalar supermultiplets in our $4 D, \mathcal{N}=1$ component notation. We will then dimensionally reduce these multiplets to the 0 -brane by considering all fields to have only time dependence. Finally, we will organize these dimensionally reduced transformation laws into Adinkras for both supermultiplets. 


\section{1 $4 D, \mathcal{N}=1$ Transformation Laws}

In component form, the real scalar supermultiplet can be written as the following set of supersymmetric transformation laws

$$
\begin{aligned}
\mathrm{D}_{a} \boldsymbol{K} & =\boldsymbol{\zeta}_{a} \\
\mathrm{D}_{a} \boldsymbol{\zeta}_{b} & =i\left(\gamma^{\mu}\right)_{a b} \partial_{\mu} \boldsymbol{K}+\left(\gamma^{5} \gamma^{\mu}\right)_{a b} \boldsymbol{U}_{\mu}+i C_{a b} \boldsymbol{M}+\left(\gamma^{5}\right)_{a b} \boldsymbol{N} \\
\mathrm{D}_{a} \boldsymbol{M} & =\frac{1}{2} \boldsymbol{\Lambda}_{a}-\frac{1}{2}\left(\gamma^{\nu}\right)_{a}{ }_{a}^{d} \partial_{\nu} \boldsymbol{\zeta}_{d} \\
\mathrm{D}_{a} \boldsymbol{N} & =-i \frac{1}{2}\left(\gamma^{5}\right)_{a}{ }^{d} \boldsymbol{\Lambda}_{d}+i \frac{1}{2}\left(\gamma^{5} \gamma^{\nu}\right)_{a}{ }^{d} \partial_{\nu} \boldsymbol{\zeta}_{d} \\
\mathrm{D}_{a} \boldsymbol{U}_{\mu} & =i \frac{1}{2}\left(\gamma^{5} \gamma_{\mu}\right)_{a}{ }^{d} \boldsymbol{\Lambda}_{d}-i \frac{1}{2}\left(\gamma^{5} \gamma^{\nu} \gamma_{\mu}\right)_{a}{ }_{a} \partial_{\nu} \boldsymbol{\zeta}_{d} \\
\mathrm{D}_{a} \boldsymbol{\Lambda}_{b} & =i\left(\gamma^{\mu}\right)_{a b} \partial_{\mu} \boldsymbol{M}+\left(\gamma^{5} \gamma^{\mu}\right)_{a b} \partial_{\mu} \boldsymbol{N}+\left(\gamma^{5} \gamma^{\mu} \gamma^{\nu}\right)_{a b} \partial_{\mu} \boldsymbol{U}_{\nu}+i C_{a b} \mathbf{d} \\
\mathrm{D}_{a} \mathbf{d} & =-\left(\gamma^{\nu}\right)_{a}{ }^{d} \partial_{\nu} \boldsymbol{\Lambda}_{d} .
\end{aligned}
$$

where $\mathrm{D}_{a}$ is the superderivative alluded to in Section 1. The superderivatives satisfy the closure relation

$$
\left\{\mathrm{D}_{a}, \mathrm{D}_{b}\right\}=2 i\left(\gamma^{\mu}\right)_{a b} \partial_{\mu}
$$

for all fields in the real scalar supermultiplet (2.1).

The real pseudoscalar supermultiplet can be defined from the real scalar supermultiplet via

$$
\begin{aligned}
& \boldsymbol{K} \rightarrow \boldsymbol{L}, \quad \boldsymbol{\zeta}_{a} \rightarrow i\left(\gamma^{5}\right)_{a}{ }^{d} \boldsymbol{\rho}_{d}, \quad \boldsymbol{M} \rightarrow \widetilde{\boldsymbol{N}}, \boldsymbol{N} \rightarrow-\widetilde{\boldsymbol{M}} \\
& \boldsymbol{U}_{\mu} \rightarrow \boldsymbol{V}_{\mu}, \quad \boldsymbol{\Lambda}_{a} \rightarrow-i\left(\gamma^{5}\right)_{a}{ }^{d} \widetilde{\boldsymbol{\Lambda}}_{d}, \quad \mathbf{d} \rightarrow \widetilde{\mathbf{d}}
\end{aligned}
$$

and this yields the pseudoscalar supermultiplet $(R P S)$, which satisfies

$$
\begin{aligned}
\mathrm{D}_{a} \boldsymbol{L} & =i\left(\gamma^{5}\right)_{a}{ }^{d} \boldsymbol{\rho}_{d} \\
\mathrm{D}_{a} \boldsymbol{\rho}_{b} & =-\left(\gamma^{5} \gamma^{\mu}\right)_{a b} \partial_{\mu} \boldsymbol{L}+i\left(\gamma^{\mu}\right)_{a b} \boldsymbol{V}_{\mu}+\left(\gamma^{5}\right)_{a b} \widetilde{\boldsymbol{N}}+i C_{a b} \widetilde{\boldsymbol{M}} \\
\mathrm{D}_{a} \widetilde{\boldsymbol{N}} & =-i \frac{1}{2}\left(\gamma^{5}\right)_{a}{ }^{d} \widetilde{\boldsymbol{\Lambda}}_{d}+i \frac{1}{2}\left(\gamma^{5} \gamma^{\mu}\right)_{a}{ }^{d} \partial_{\mu} \boldsymbol{\rho}_{d} \\
\mathrm{D}_{a} \widetilde{\boldsymbol{M}} & =\frac{1}{2} \widetilde{\boldsymbol{\Lambda}}_{a}-\frac{1}{2}\left(\gamma^{\mu}\right)_{a}{ }^{d} \partial_{\mu} \boldsymbol{\rho}_{d} \\
\mathrm{D}_{a} \boldsymbol{V}_{\mu} & =-\frac{1}{2}\left(\gamma_{\mu}\right)_{a}{ }^{d} \widetilde{\boldsymbol{\Lambda}}_{d}+\frac{1}{2}\left(\gamma^{\nu} \gamma_{\mu}\right)_{a}{ }_{a}{ } \partial_{\nu} \boldsymbol{\rho}_{d} \\
\mathrm{D}_{a} \widetilde{\boldsymbol{\Lambda}}_{b} & =\left(\gamma^{5} \gamma^{\mu}\right)_{a b} \partial_{\mu} \widetilde{\boldsymbol{N}}+i\left(\gamma^{\mu}\right)_{a b} \partial_{\mu} \widetilde{\boldsymbol{M}}+i\left(\gamma^{\mu} \gamma^{\nu}\right)_{a b} \partial_{\mu} \boldsymbol{V}_{\nu}-\left(\gamma^{5}\right)_{a b} \widetilde{\mathbf{d}} \\
\mathrm{D}_{a} \widetilde{\mathbf{d}} & =-i\left(\gamma^{5} \gamma^{\mu}\right)_{a}{ }^{d} \partial_{\mu} \widetilde{\boldsymbol{\Lambda}}_{d} .
\end{aligned}
$$

where the superderivatives again satisfy the closure relation (2.2) for all fields in the real pseudoscalar supermultiplet (2.4).

\subsection{Dimensional Reduction and Adinkras}

Performing the one-dimensional reduction of the real scalar supermultiplet (2.1) and defining $\boldsymbol{X}_{I J}=$ $-\boldsymbol{X}_{J I} \equiv-i \frac{1}{2} \mathrm{D}_{[I} \mathrm{D}_{J]} \boldsymbol{K}=-i \frac{1}{2}\left(\mathrm{D}_{I} \mathrm{D}_{J}-\mathrm{D}_{J} \mathrm{D}_{I}\right) \boldsymbol{K}$ we find:

$$
\begin{array}{lll}
\boldsymbol{X}_{12} \equiv \boldsymbol{U}_{2}-\boldsymbol{M}, & \boldsymbol{X}_{13} \equiv \boldsymbol{U}_{3}-\boldsymbol{N}, & \boldsymbol{X}_{23} \equiv \boldsymbol{U}_{1}-\boldsymbol{U}_{0} \\
\boldsymbol{X}_{14} \equiv \boldsymbol{U}_{0}+\boldsymbol{U}_{1}, & \boldsymbol{X}_{24} \equiv-\boldsymbol{U}_{3}-\boldsymbol{N}, & \boldsymbol{X}_{34} \equiv \boldsymbol{U}_{2}+\boldsymbol{M}
\end{array}
$$


Table 1: The superdifferential relations between the one-dimensionally reduced components of the real scalar superfield.

\begin{tabular}{cc|cccc|cccccc|ccccc|c} 
& $\boldsymbol{K}$ & $\boldsymbol{\zeta}_{1}$ & $\boldsymbol{\zeta}_{2}$ & $\boldsymbol{\zeta}_{3}$ & $\boldsymbol{\zeta}_{4}$ & $\boldsymbol{X}_{12}$ & $\boldsymbol{X}_{13}$ & $\boldsymbol{X}_{14}$ & $\boldsymbol{X}_{23}$ & $\boldsymbol{X}_{24}$ & $\boldsymbol{X}_{34}$ & $-\boldsymbol{\Lambda}_{2}$ & $\boldsymbol{\Lambda}_{1}$ & $\boldsymbol{\Lambda}_{4}$ & $-\boldsymbol{\Lambda}_{3}$ & $\mathbf{d}$ \\
\hline $\mathrm{D}_{1}:$ & $\boldsymbol{\zeta}_{1}$ & $i \dot{\boldsymbol{K}}$ & $i \boldsymbol{X}_{12}$ & $i \boldsymbol{X}_{13}$ & $i \boldsymbol{X}_{14}$ & $\dot{\boldsymbol{\zeta}}_{2}$ & $\dot{\boldsymbol{\zeta}}_{3}$ & $\dot{\boldsymbol{\zeta}}_{4}$ & $-\boldsymbol{\Lambda}_{3}$ & $-\boldsymbol{\Lambda}_{4}$ & $\boldsymbol{\Lambda}_{1}$ & $i \mathbf{d}$ & $i \dot{\boldsymbol{X}}_{34}$ & $-i \dot{\boldsymbol{X}}_{24}$ & $i \dot{\boldsymbol{X}}_{23}$ & $-\dot{\boldsymbol{\Lambda}}_{2}$ \\
$\mathrm{D}_{2}:$ & $\boldsymbol{\zeta}_{2}$ & $-i \boldsymbol{X}_{12}$ & $i \dot{\boldsymbol{K}}$ & $i \boldsymbol{X}_{23}$ & $i \boldsymbol{X}_{24}$ & $-\dot{\boldsymbol{\zeta}}_{1}$ & $\boldsymbol{\Lambda}_{3}$ & $\boldsymbol{\Lambda}_{4}$ & $\dot{\boldsymbol{\zeta}}_{3}$ & $\dot{\boldsymbol{\zeta}}_{4}$ & $\boldsymbol{\Lambda}_{2}$ & $-i \dot{\boldsymbol{X}}_{34}$ & $i \mathbf{d}$ & $i \dot{\boldsymbol{X}}_{14}$ & $-i \dot{\boldsymbol{X}}_{13}$ & $\dot{\boldsymbol{\Lambda}}_{1}$ \\
$\mathrm{D}_{3}:$ & $\boldsymbol{\zeta}_{3}$ & $-i \boldsymbol{X}_{13}$ & $-i \boldsymbol{X}_{23}$ & $i \dot{\boldsymbol{K}}$ & $i \boldsymbol{X}_{34}$ & $-\boldsymbol{\Lambda}_{3}$ & $-\dot{\boldsymbol{\zeta}}_{1}$ & $-\boldsymbol{\Lambda}_{1}$ & $-\dot{\boldsymbol{\zeta}}_{2}$ & $-\boldsymbol{\Lambda}_{2}$ & $\dot{\boldsymbol{\zeta}}_{4}$ & $i \dot{\boldsymbol{X}}_{24}$ & $-i \dot{\boldsymbol{X}}_{14}$ & $i \mathbf{d}$ & $i \dot{\boldsymbol{X}}_{12}$ & $\dot{\boldsymbol{\Lambda}}_{4}$ \\
$\mathrm{D}_{4}:$ & $\boldsymbol{\zeta}_{4}$ & $-i \boldsymbol{X}_{14}$ & $-i \boldsymbol{X}_{24}$ & $-i \boldsymbol{X}_{34}$ & $i \dot{\boldsymbol{K}}$ & $-\boldsymbol{\Lambda}_{4}$ & $\boldsymbol{\Lambda}_{1}$ & $-\dot{\boldsymbol{\zeta}}_{1}$ & $\boldsymbol{\Lambda}_{2}$ & $-\dot{\boldsymbol{\zeta}}_{2}$ & $-\dot{\boldsymbol{\zeta}}_{3}$ & $-i \dot{\boldsymbol{X}}_{23}$ & $i \dot{\boldsymbol{X}}_{13}$ & $-i \dot{\boldsymbol{X}}_{12}$ & $i \mathbf{d}$ & $-\dot{\boldsymbol{\Lambda}}_{3}$ \\
\hline
\end{tabular}

we can write the transformation laws as in the Table 1 . With the conventions of Refs. $[17,18]$, we can depict the dimensionally reduced transformation laws for the real scalar supermultiplet $(R S S)$ as the Adinkra in (2.6)

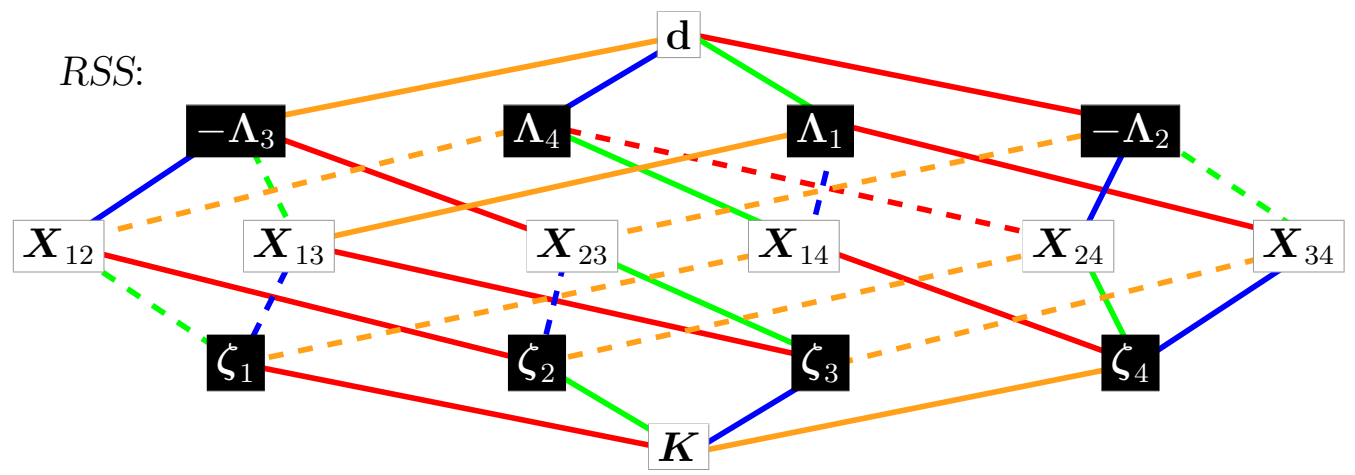

where e.g., $\mathrm{D}_{1} \boldsymbol{K}=\boldsymbol{\zeta}_{1}$ is depicted by the solid red edge, and $\mathrm{D}_{1} \boldsymbol{X}_{24}=-\boldsymbol{\Lambda}_{4}$ by the dashed red edge where the dashing encodes the minus sign; following an edge downward results in the $\tau$-derivative of the indicated component field. Owing to the factors of $i$ in all fermion to boson transformations in Table 1, the $\boldsymbol{\zeta}_{2}-\boldsymbol{X}_{12}$ solid red edge depicts for instance the relation $\mathrm{D}_{1} \boldsymbol{\zeta}_{2}=i \boldsymbol{X}_{12}$.

With the substitutions (2.3), written more explicitly as in Eq. (2.7)

$$
\begin{gathered}
\boldsymbol{K} \rightarrow \boldsymbol{L}, \quad \boldsymbol{M} \rightarrow \widetilde{\boldsymbol{N}}, \quad \boldsymbol{N} \rightarrow-\widetilde{\boldsymbol{M}} \\
U_{\mu} \rightarrow V_{\mu}, \quad \mathrm{d} \rightarrow \widetilde{\mathrm{d}} \\
\boldsymbol{\zeta}_{1} \rightarrow-\boldsymbol{\rho}_{4}, \quad \boldsymbol{\zeta}_{2} \rightarrow \boldsymbol{\rho}_{3}, \quad \boldsymbol{\zeta}_{3} \rightarrow-\boldsymbol{\rho}_{2}, \quad \boldsymbol{\zeta}_{4} \rightarrow \boldsymbol{\rho}_{1} \\
\Lambda_{1} \rightarrow \widetilde{\Lambda}_{4}, \quad \Lambda_{2} \rightarrow-\widetilde{\Lambda}_{3}, \quad \Lambda_{3} \rightarrow \widetilde{\Lambda}_{2}, \quad \Lambda_{4} \rightarrow-\widetilde{\Lambda}_{1},
\end{gathered}
$$

and the definitions $\boldsymbol{Y}_{I J}=-\boldsymbol{Y}_{J I} \equiv-i \frac{1}{2} \mathrm{D}_{[I} \mathrm{D}_{J]} \boldsymbol{L}$ :

$$
\begin{array}{lll}
\boldsymbol{Y}_{12} \equiv \boldsymbol{V}_{2}-\widetilde{\boldsymbol{N}} & , \boldsymbol{Y}_{13} \equiv \boldsymbol{V}_{3}+\widetilde{\boldsymbol{M}}, & \boldsymbol{Y}_{23} \equiv \boldsymbol{V}_{1}-\boldsymbol{V}_{0} \\
\boldsymbol{Y}_{14} \equiv \boldsymbol{V}_{0}+\boldsymbol{V}_{1}, & \boldsymbol{Y}_{24} \equiv \widetilde{\boldsymbol{M}}-\boldsymbol{V}_{3}, & \boldsymbol{Y}_{34} \equiv \boldsymbol{V}_{2}+\widetilde{\boldsymbol{N}}
\end{array}
$$

Table 1 transforms into Table 2 for the real pseudoscalar supermultiplet (2.4) which can then be depicted succinctly as the Adinkra in (2.9). 
Table 2: The superdifferential relations between the one-dimensionally reduced components of the real pseudoscalar superfield.

\begin{tabular}{cc|cccc|cccccc|cccc|c} 
& $\boldsymbol{L}$ & $-\boldsymbol{\rho}_{4}$ & $\boldsymbol{\rho}_{3}$ & $-\boldsymbol{\rho}_{2}$ & $\boldsymbol{\rho}_{1}$ & $\boldsymbol{Y}_{12}$ & $\boldsymbol{Y}_{13}$ & $\boldsymbol{Y}_{14}$ & $\boldsymbol{Y}_{23}$ & $\boldsymbol{Y}_{24}$ & $\boldsymbol{Y}_{34}$ & $\widetilde{\boldsymbol{\Lambda}}_{3}$ & $\widetilde{\boldsymbol{\Lambda}}_{4}$ & $-\widetilde{\boldsymbol{\Lambda}}_{1}$ & $-\widetilde{\boldsymbol{\Lambda}}_{2}$ & $\widetilde{\mathbf{d}}$ \\
\hline $\mathrm{D}_{1}:$ & $-\boldsymbol{\rho}_{4}$ & $i \dot{\boldsymbol{L}}$ & $i \boldsymbol{Y}_{12}$ & $i \boldsymbol{Y}_{13}$ & $i \boldsymbol{Y}_{14}$ & $\dot{\boldsymbol{\rho}}_{3}$ & $-\dot{\boldsymbol{\rho}}_{2}$ & $\dot{\boldsymbol{\rho}}_{1}$ & $-\widetilde{\boldsymbol{\Lambda}}_{2}$ & $\widetilde{\boldsymbol{\Lambda}}_{1}$ & $\widetilde{\boldsymbol{\Lambda}}_{4}$ & $i \widetilde{\mathbf{d}}$ & $i \dot{\boldsymbol{Y}}_{34}$ & $-i \dot{\boldsymbol{Y}}_{24}$ & $i \dot{\boldsymbol{Y}}_{23}$ & $\dot{\tilde{\boldsymbol{\Lambda}}}_{3}$ \\
$\mathrm{D}_{2}:$ & $\boldsymbol{\rho}_{3}$ & $-i \boldsymbol{Y}_{12}$ & $i \dot{\boldsymbol{L}}$ & $i \boldsymbol{Y}_{23}$ & $i \boldsymbol{Y}_{24}$ & $\dot{\boldsymbol{\rho}}_{4}$ & $\widetilde{\boldsymbol{\Lambda}}_{2}$ & $-\widetilde{\boldsymbol{\Lambda}}_{1}$ & $-\dot{\boldsymbol{\rho}}_{2}$ & $\dot{\boldsymbol{\rho}}_{1}$ & $-\widetilde{\boldsymbol{\Lambda}}_{3}$ & $-i \dot{\boldsymbol{Y}}_{34}$ & $i \widetilde{\mathbf{d}}$ & $i \dot{\boldsymbol{Y}}_{14}$ & $-i \dot{\boldsymbol{Y}}_{13}$ & $\dot{\tilde{\boldsymbol{\Lambda}}}_{4}$ \\
$\mathrm{D}_{3}:$ & $-\boldsymbol{\rho}_{2}$ & $-i \boldsymbol{Y}_{13}$ & $-i \boldsymbol{Y}_{23}$ & $i \dot{\boldsymbol{L}}$ & $i \boldsymbol{Y}_{34}$ & $-\widetilde{\boldsymbol{\Lambda}}_{2}$ & $\dot{\boldsymbol{\rho}}_{4}$ & $-\widetilde{\boldsymbol{\Lambda}}_{4}$ & $-\dot{\boldsymbol{\rho}}_{3}$ & $\widetilde{\boldsymbol{\Lambda}}_{3}$ & $\dot{\boldsymbol{\rho}}_{1}$ & $i \dot{\boldsymbol{Y}}_{24}$ & $-i \dot{\boldsymbol{Y}}_{14}$ & $i \widetilde{\mathbf{d}}$ & $i \dot{\boldsymbol{Y}}_{12}$ & $-\dot{\tilde{\boldsymbol{\Lambda}}}_{1}$ \\
$\mathrm{D}_{4}:$ & $\boldsymbol{\rho}_{1}$ & $-i \boldsymbol{Y}_{14}$ & $-i \boldsymbol{Y}_{24}$ & $-i \boldsymbol{Y}_{34}$ & $i \dot{\boldsymbol{L}}$ & $\widetilde{\boldsymbol{\Lambda}}_{1}$ & $\widetilde{\boldsymbol{\Lambda}}_{4}$ & $\dot{\boldsymbol{\rho}}_{4}$ & $-\widetilde{\boldsymbol{\Lambda}}_{3}$ & $-\dot{\boldsymbol{\rho}}_{3}$ & $\dot{\boldsymbol{\rho}}_{2}$ & $-i \dot{\boldsymbol{Y}}_{23}$ & $i \dot{\boldsymbol{Y}}_{13}$ & $-i \dot{\boldsymbol{Y}}_{12}$ & $i \widetilde{\mathbf{d}}$ & $-\dot{\widetilde{\boldsymbol{\Lambda}}}_{2}$ \\
\hline
\end{tabular}

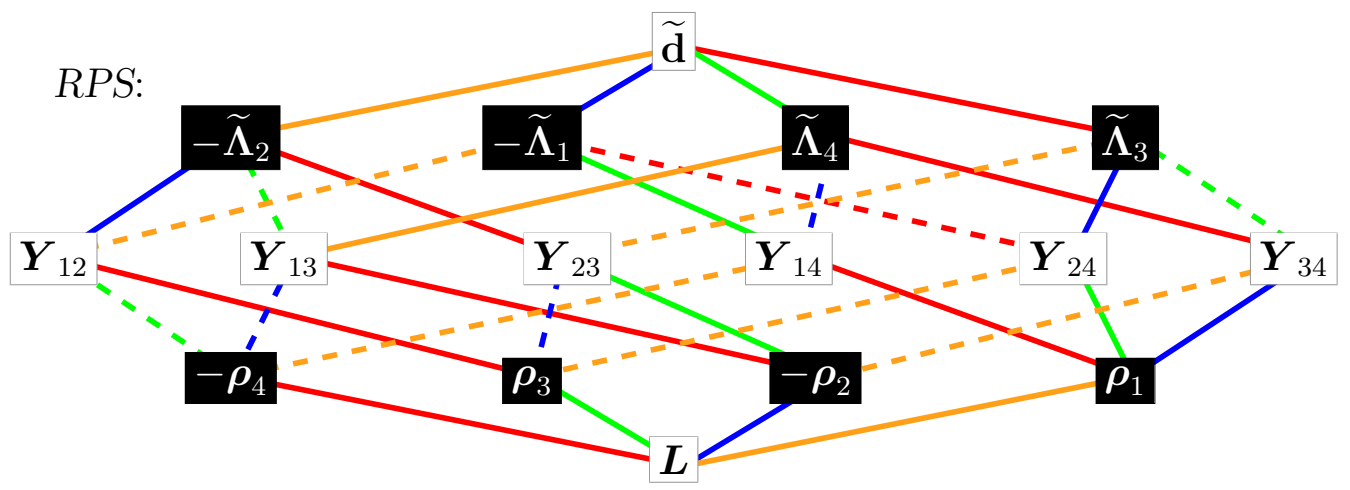

\section{The Complex Linear Supermultiplet}

\subsection{The Complex Linear Supermultiplet Fusion}

In the introduction, the complex linear supermultiplet was called a "red-headed stepchild." The reason for this can be seen by reviewing its history. The proper appearance of the complex linear supermultiplet occurred in works on the subjects of superfield supergravity [19] and the use of superspace [14]. The first comment that it could be related by a duality transformation to the usual chiral multiplet was contained in Ref. [20] where it was noted that Zumino had previously given a first-order action appropriate for this purpose [21]. In 1984, it was proposed [22] that the existence of the chiral supermultiplet and the complex linear supermultiplet offered the possibility of assigning left-handed and right-handed fermions to very distinct supersymmetry representations with one handedness being described by chiral supermultiplet and the other handedness to arise from the complex linear supermultiplet. Since that time, one of the authors (SJG) has returned to investigate the complex linear supermultiplet many times in applications such as phenomenological actions [23,24], six dimensional SUSY sigma-models [25,26], 4D $\mathcal{N}=2$ SUSY sigma-models [27, $28,29]$, and in relation to a fundamental representation theory [30,31]. In recent times, principally due to S. Kuzenko and collaborators, there have appeared many new and impressive results [32, $33,34,35,36,37,38,39,40,41,42,43,44,45,46,47]$ that lend broad and deep insights into the use of the complex linear multiplet in additional topics such as Goldstone superfields, sigma-models, and their relation to harmonic and projective superspace models.

So by this point, the "red-headed stepchild" has been shown to provide a wide array of applications. There are even some hints $[48,49]$ that the complex linear supermultiplet may be of interest 
for effective string theories.

We can construct the complex linear supermultiplet from fusion of a real scalar and pseudoscalar supermultiplet with the constraint

$$
\left(C^{a b}+\left(\gamma^{5}\right)^{a b}\right) \mathrm{D}_{a} \mathrm{D}_{b}(\boldsymbol{K}+i \boldsymbol{L})=0 .
$$

As $C^{a b}$ is purely real and $\left(\gamma^{5}\right)^{a b}$ is purely imaginary, we can split this constraint into its real and imaginary parts

$$
\begin{array}{lll}
\mathrm{D}_{a} \mathrm{D}_{b}\left(C^{a b} \boldsymbol{K}+i\left(\gamma^{5}\right)^{a b} \boldsymbol{L}\right)=0 & \Rightarrow & \left(\mathrm{D}_{[3} \mathrm{D}_{4]}-\mathrm{D}_{[1} \mathrm{D}_{2]}\right) \boldsymbol{K}=\left(\mathrm{D}_{[1} \mathrm{D}_{3]}+\mathrm{D}_{[2} \mathrm{D}_{4]}\right) \boldsymbol{L} \\
\mathrm{D}_{a} \mathrm{D}_{b}\left(\left(\gamma^{5}\right)^{a b} \boldsymbol{K}+i C^{a b} \boldsymbol{L}\right)=0 & \Rightarrow & \left(\mathrm{D}_{[1} \mathrm{D}_{3]}+\mathrm{D}_{[2} \mathrm{D}_{4]}\right) \boldsymbol{K}=-\left(\mathrm{D}_{[3} \mathrm{D}_{4]}-\mathrm{D}_{[1} \mathrm{D}_{2]}\right) \boldsymbol{L}
\end{array}
$$

which result in the following simple constraints:

$$
\begin{gathered}
\phi_{1} \equiv \boldsymbol{M}-\widetilde{\boldsymbol{M}}=0 \Rightarrow \boldsymbol{Y}_{13}+\boldsymbol{Y}_{24}=\boldsymbol{X}_{34}-\boldsymbol{X}_{12} \\
\phi_{2} \equiv \boldsymbol{N}-\widetilde{\boldsymbol{N}}=0 \Rightarrow \boldsymbol{X}_{13}+\boldsymbol{X}_{24}=\boldsymbol{Y}_{12}-\boldsymbol{Y}_{34}
\end{gathered}
$$

After calculating either of

$$
\mathrm{D}_{a} \boldsymbol{\phi}_{1}=0 \quad \text { or } \quad \mathrm{D}_{a} \boldsymbol{\phi}_{2}=0
$$

we uncover the following constraints on the fermions

$$
\boldsymbol{\Psi}_{a} \equiv \boldsymbol{\Lambda}_{a}-\widetilde{\boldsymbol{\Lambda}}_{a}-\left(\gamma^{\mu}\right)_{a}^{d} \partial_{\mu}\left(\boldsymbol{\zeta}_{d}-\boldsymbol{\rho}_{d}\right)=0
$$

Defining

$$
2 \boldsymbol{\beta}_{a} \equiv \boldsymbol{\Lambda}_{a}+\widetilde{\boldsymbol{\Lambda}}_{a}-\left(\gamma^{\mu}\right)_{a}^{b} \partial_{\mu}\left(\boldsymbol{\zeta}_{b}+\boldsymbol{\rho}_{b}\right)
$$

we next eliminate $\boldsymbol{\Lambda}_{a}$ and $\widetilde{\boldsymbol{\Lambda}}_{a}$ from the system by solving for them in terms of $\widetilde{\boldsymbol{\beta}}_{a}$

$$
\begin{aligned}
& \boldsymbol{\Lambda}_{a}=\boldsymbol{\beta}_{a}+\left(\gamma^{\mu}\right)_{a}{ }^{d} \partial_{\mu} \boldsymbol{\zeta}_{d} \\
& \widetilde{\boldsymbol{\Lambda}}_{a}=\boldsymbol{\beta}_{a}+\left(\gamma^{\mu}\right)_{a}{ }^{d} \partial_{\mu} \boldsymbol{\rho}_{d} .
\end{aligned}
$$

Our final two constraints are

$$
\begin{array}{rll}
\left(\gamma^{5}\right)^{a b} \mathrm{D}_{a} \mathrm{D}_{b} \boldsymbol{\phi}_{1}=0 & \text { or } \quad C^{a b} \mathrm{D}_{a} \mathrm{D}_{b} \boldsymbol{\phi}_{2}=0 \quad \Rightarrow \quad \boldsymbol{\Phi}_{1} \equiv \widetilde{\mathbf{d}}+2 \partial_{\mu} \boldsymbol{U}^{\mu}+\square \boldsymbol{L}=0 \\
C^{a b} \mathrm{D}_{a} \mathrm{D}_{b} \boldsymbol{\phi}_{1}=0 & \text { or } \quad\left(\gamma^{5}\right)^{a b} \mathrm{D}_{a} \mathrm{D}_{b} \boldsymbol{\phi}_{2}=0 \quad \Rightarrow \quad \boldsymbol{\Phi}_{2} \equiv \mathbf{d}-2 \partial_{\mu} \boldsymbol{V}^{\mu}+\square \boldsymbol{K}=0
\end{array}
$$

with $\square \equiv \partial_{\mu} \partial^{\mu}=\eta^{\mu \nu} \partial_{\mu} \partial_{\nu}$. This leaves a supermultiplet parametrized by the remaining $(2|8| 10|4| 0)$ component fields:

$$
\left(\boldsymbol{K}, \boldsymbol{L}\left|\boldsymbol{\zeta}_{a}, \boldsymbol{\rho}_{b}\right| \boldsymbol{M}, \boldsymbol{N}, \boldsymbol{U}_{\mu}, \boldsymbol{V}_{\nu} \mid \boldsymbol{\beta}_{a}\right)
$$

Owing to the component field substitutions (3.7) and (3.8), these $12+12$ component fields indeed span the complete supermultiplet, reduced from the initial $16+16$ component fields by means of the constraints (3.3). The transformation laws of the resulting $12+12$ component complex linear superfield are as in Eq. (3.10).

$$
\mathrm{D}_{a} \boldsymbol{K}=\boldsymbol{\zeta}_{a}
$$




$$
\begin{aligned}
\mathrm{D}_{a} \boldsymbol{L} & =i\left(\gamma^{5}\right)_{a}^{b} \boldsymbol{\rho}_{b} \\
\mathrm{D}_{a} \boldsymbol{\rho}_{b} & =i C_{a b} \boldsymbol{M}+\left(\gamma^{5}\right)_{a b} \boldsymbol{N}+i\left(\gamma^{\mu}\right)_{a b} \boldsymbol{V}_{\mu}-\left(\gamma^{5} \gamma^{\mu}\right)_{a b} \partial_{\mu} \boldsymbol{L} \\
\mathrm{D}_{a} \boldsymbol{\zeta}_{b} & =i C_{a b} \boldsymbol{M}+\left(\gamma^{5}\right)_{a b} \boldsymbol{N}+\left(\gamma^{5} \gamma^{\mu}\right)_{a b} \boldsymbol{U}_{\mu}+i\left(\gamma^{\mu}\right)_{a b} \partial_{\mu} \boldsymbol{K} \\
\mathrm{D}_{a} \boldsymbol{M} & =\frac{1}{2} \boldsymbol{\beta}_{a} \\
\mathrm{D}_{a} \boldsymbol{N} & =-i \frac{1}{2}\left(\gamma^{5}\right)_{a}^{b} \boldsymbol{\beta}_{b} \\
\mathrm{D}_{a} \boldsymbol{U}_{\mu} & =i \frac{1}{2}\left(\gamma^{5} \gamma_{\mu}\right)_{a}^{b} \boldsymbol{\beta}_{b}-i \frac{1}{2}\left(\gamma^{5}\left[\gamma^{\nu}, \gamma_{\mu}\right]\right)_{a}^{b} \partial_{\nu} \boldsymbol{\zeta}_{b} \\
\mathrm{D}_{a} \boldsymbol{V}_{\mu} & =-\frac{1}{2}\left(\gamma_{\mu}\right)_{a}{ }^{b} \boldsymbol{\beta}_{b}+\frac{1}{2}\left(\left[\gamma^{\nu}, \gamma_{\mu}\right]\right)_{a}{ }^{b} \partial_{\nu} \boldsymbol{\rho}_{b} \\
\mathrm{D}_{a} \boldsymbol{\beta}_{b} & =2 i\left(\gamma^{\mu}\right)_{a b} \partial_{\mu} \boldsymbol{M}+2\left(\gamma^{5} \gamma^{\mu}\right)_{a b} \partial_{\mu} \boldsymbol{N}+2\left(\gamma^{5}\right)_{a b} \partial^{\mu} \boldsymbol{U}_{\mu}+2 i C_{a b} \partial^{\mu} \boldsymbol{V}_{\mu} .
\end{aligned}
$$

Noting that the redefinitions (3.7) and (3.8) indicate (1) "1-several" component field replacements and (2) include second derivatives, the reader might suspect the latter of these to imply a violation of the Haag-Łopusański-Sohnius theorem in that a supercharge might transform a component field into another one the engineering dimension of which differs from the original one by more than $\pm \frac{1}{2}$. In fact, the (standard [14,50] and) manifestly supersymmetric complex superfield formulation and treatment of $C L S$ reassures us that this is not the case, and the appearance of the second derivatives merely points to the inadequacy of the basis (3.9).

In turn, we show that the former of these features implies that the CLS is not adinkraic; this result may simply herald the "inconvenient truth:" that most ${ }^{2}$ representations of supersymmetry are not adinkraic. This agrees with the recent result of Ref. [51].

As was noted in [31], the constraints in (3.3), (3.5), and (3.8)

$$
\left(\phi_{1}, \phi_{2}\left|\Psi_{a}\right| \Phi_{1}, \Phi_{2}\right)
$$

where

$$
\begin{aligned}
& \phi_{1} \equiv \boldsymbol{M}-\widetilde{\boldsymbol{M}} \quad, \quad \phi_{2} \equiv \boldsymbol{N}-\widetilde{\boldsymbol{N}}, \\
& \boldsymbol{\Psi}_{a} \equiv \boldsymbol{\Lambda}_{a}-\widetilde{\boldsymbol{\Lambda}}_{a}-\left(\gamma^{\mu}\right)_{a}{ }^{d} \partial_{\mu}\left(\boldsymbol{\zeta}_{d}-\boldsymbol{\rho}_{d}\right) \\
& \boldsymbol{\Phi}_{1} \equiv \widetilde{\mathbf{d}}+2 \partial_{\mu} \boldsymbol{U}^{\mu}+\square \boldsymbol{L} \quad, \quad \boldsymbol{\Phi}_{2} \equiv \mathbf{d}-2 \partial_{\mu} \boldsymbol{V}^{\mu}+\square \boldsymbol{K}
\end{aligned}
$$

themselves form a chiral supermultiplet. Thus, if we draw the Adinkras for the RSS and the RPS the imposition of the constraints can be indicated by the insertion of the Adinkra of a chiral multiplet relating to the components of the RSS and the RPS which is then set to zero. This chiral multiplet is illustrated in the 'blue region' of Fig. 1 where the rightmost Adinkra corresponds to the fields and transformation laws in Eq. (3.10). Figure 1 is a 'blueprint' for the CLS Adinkra with explicit field content which we will build in Section 3.2. The process depicted in Fig. 1 was called 'zippering' in the work of Ref. [52]. However, K. Iga has noted that the process imposed on the Adinkra in someways resembles the process of constructing a gnomon [53] in classical geometry.

\footnotetext{
${ }^{2}$ As the "1-several" component field replacements (3.7) and (3.8) stem from the simplicity of the superdifferential constraints (3.3) and the somewhat obvious fact that there exists indefinitely more non-simple superdifferential constraints [15], the subset of simple superdifferential constraints and so-defined simple supermultiplets is of measure zero in the totality of all superdifferential constraints and so-defined supermultiplets.
} 


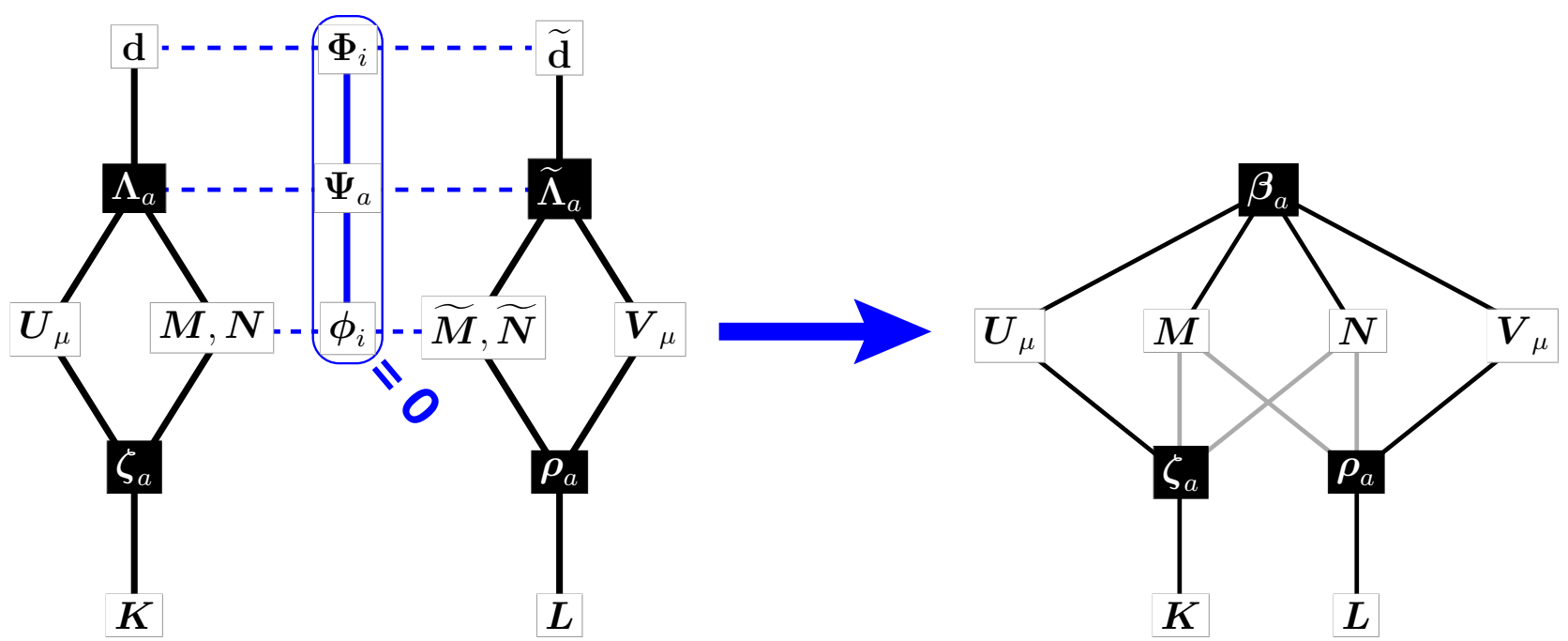

Figure 1: A schematic of the 'zippering' process that fuses the RSS and RPS (left) into the CLS (right). The black edges indicate bi-directional transformations and the grey edges indicate uni-directional transformations. For instance, the black edge $\boldsymbol{M}-\boldsymbol{\beta}_{a}$ indicates that $\mathrm{D}_{a} M \supset \boldsymbol{\beta}_{a}$ and $\mathrm{D}_{a} \boldsymbol{\beta}_{b} \supset \boldsymbol{M}$, where as the grey edge $\boldsymbol{M}-\boldsymbol{\zeta}_{a}$ indicates that, e.g., $\mathrm{D}_{a} \boldsymbol{\zeta}_{b} \supset \boldsymbol{M}$ but $\mathrm{D}_{a} \boldsymbol{M} \not \supset \boldsymbol{\zeta}_{a}$.

\subsection{O-brane Reduction and Adinkra}

The two constraints (3.3) allow us to remove $X_{13}$ and $Y_{13}$ from the resulting dimensionally reduced complex linear supermultiplet, leaving us with the linearly independent set:

$$
\left(\boldsymbol{K}, \boldsymbol{L}\left|\boldsymbol{\zeta}_{I}, \boldsymbol{\rho}_{J}\right| \boldsymbol{X}_{12}, \boldsymbol{X}_{14}, \boldsymbol{X}_{23}, \boldsymbol{X}_{24}, \boldsymbol{X}_{34}, \boldsymbol{Y}_{12}, \boldsymbol{Y}_{14}, \boldsymbol{Y}_{23}, \boldsymbol{Y}_{24}, \boldsymbol{Y}_{34} \mid \boldsymbol{\beta}_{I}\right)
$$

where

$$
\begin{aligned}
\boldsymbol{X}_{12}= & \boldsymbol{U}_{2}-\boldsymbol{M}, \quad \boldsymbol{X}_{23}=\boldsymbol{U}_{1}-\boldsymbol{U}_{0}, \quad \boldsymbol{X}_{24}=-\boldsymbol{U}_{3}-\boldsymbol{N}, \\
& \boldsymbol{X}_{14}=\boldsymbol{U}_{0}+\boldsymbol{U}_{1}, \quad \boldsymbol{X}_{34}=\boldsymbol{U}_{2}+\boldsymbol{M}, \\
\boldsymbol{Y}_{12}= & \boldsymbol{V}_{2}-\boldsymbol{N}, \quad \boldsymbol{Y}_{23}=\boldsymbol{V}_{1}-\boldsymbol{V}_{0}, \quad \boldsymbol{Y}_{24}=\boldsymbol{M}-\boldsymbol{V}_{3} \\
& \boldsymbol{Y}_{14}=\boldsymbol{V}_{0}+\boldsymbol{V}_{1}, \quad \boldsymbol{Y}_{34}=\boldsymbol{V}_{2}+\boldsymbol{N}
\end{aligned}
$$

The dimensionally reduced versions of the substitutions (3.7) and (3.8) which take us from RSS and RPS union to CLS are

$$
\begin{aligned}
& \boldsymbol{\Lambda}_{1}=\boldsymbol{\beta}_{1}+\dot{\boldsymbol{\zeta}}_{2} \quad, \quad \boldsymbol{\Lambda}_{2}=\boldsymbol{\beta}_{2}-\dot{\boldsymbol{\zeta}}_{1} \quad, \quad \boldsymbol{\Lambda}_{3}=\boldsymbol{\beta}_{3}-\dot{\boldsymbol{\zeta}}_{4} \quad, \quad \boldsymbol{\Lambda}_{4}=\boldsymbol{\beta}_{4}+\dot{\boldsymbol{\zeta}}_{3}, \\
& \widetilde{\boldsymbol{\Lambda}}_{1}=\boldsymbol{\beta}_{1}+\dot{\boldsymbol{\rho}}_{2} \quad, \quad \widetilde{\boldsymbol{\Lambda}}_{2}=\boldsymbol{\beta}_{2}-\dot{\boldsymbol{\rho}}_{1} \quad, \quad \widetilde{\boldsymbol{\Lambda}}_{3}=\boldsymbol{\beta}_{3}-\dot{\boldsymbol{\rho}}_{4} \quad, \quad \widetilde{\boldsymbol{\Lambda}}_{4}=\boldsymbol{\beta}_{4}+\dot{\boldsymbol{\rho}}_{3}, \\
& \mathbf{d}=-2 \dot{\boldsymbol{V}}_{0}+\ddot{\boldsymbol{K}}=\dot{\boldsymbol{Y}}_{23}-\dot{\boldsymbol{Y}}_{14}+\ddot{\boldsymbol{K}} \quad, \quad \widetilde{\mathbf{d}}=2 \dot{\boldsymbol{U}}_{0}+\ddot{\boldsymbol{L}}=\dot{\boldsymbol{X}}_{14}-\dot{\boldsymbol{X}}_{23}+\ddot{\boldsymbol{L}}
\end{aligned}
$$

We now copy the dimensionally reduced transformation laws in Tables 1and 2 for the real scalar and pseudoscalar supermultiplets, respectively (utilizing the substitutions (2.7)), and perform the substitutions (3.3), (3.14), and (3.15), omitting also the substituted fields, $\boldsymbol{X}_{13}, \boldsymbol{Y}_{13}, \boldsymbol{\Lambda}_{I}, \widetilde{\Lambda}_{J}, \mathbf{d}$ and $\widetilde{\mathbf{d}}$, from the so-obtained Table 3 . 
Table 3: The dimensionally reduced complex linear supermultiplet supersymmetry transformation rules: the initial basis (3.9).

\begin{tabular}{|c|c|c|c|c|}
\hline & $\mathrm{D}_{1}$ & $\mathrm{D}_{2}$ & $\mathrm{D}_{3}$ & $\mathrm{D}_{4}$ \\
\hline$K$ & $\zeta_{1}$ & $\zeta_{2}$ & $\zeta_{3}$ & $\zeta_{4}$ \\
\hline$L$ & $-\boldsymbol{\rho}_{4}$ & $\rho_{3}$ & $-\rho_{2}$ & $\rho_{1}$ \\
\hline$\zeta_{1}$ & $i \dot{K}$ & $-i \boldsymbol{X}_{12}$ & $i\left(\boldsymbol{X}_{24}-\boldsymbol{Y}_{12}+\boldsymbol{Y}_{34}\right)$ & $-i \boldsymbol{X}_{14}$ \\
\hline$\zeta_{2}$ & $i \boldsymbol{X}_{12}$ & $i \dot{\boldsymbol{K}}$ & $-i \boldsymbol{X}_{23}$ & $-i \boldsymbol{X}_{24}$ \\
\hline$\zeta_{3}$ & $-i\left(\boldsymbol{X}_{24}-\boldsymbol{Y}_{12}+\boldsymbol{Y}_{34}\right)$ & $i \boldsymbol{X}_{23}$ & $i \dot{\boldsymbol{K}}$ & $-i \boldsymbol{X}_{34}$ \\
\hline$\zeta_{4}$ & $i \boldsymbol{X}_{14}$ & $i \boldsymbol{X}_{24}$ & $i \boldsymbol{X}_{34}$ & $i \dot{\boldsymbol{K}}$ \\
\hline$\rho_{1}$ & $i \boldsymbol{Y}_{14}$ & $i \boldsymbol{Y}_{24}$ & $i \boldsymbol{Y}_{34}$ & $i \dot{\boldsymbol{L}}$ \\
\hline$\rho_{2}$ & $i\left(\boldsymbol{Y}_{24}+\boldsymbol{X}_{12}-\boldsymbol{X}_{34}\right)$ & $-i \boldsymbol{Y}_{23}$ & $-i \dot{\boldsymbol{L}}$ & $i \boldsymbol{Y}_{34}$ \\
\hline$\rho_{3}$ & $i \boldsymbol{Y}_{12}$ & $i \dot{\boldsymbol{L}}$ & $-i \boldsymbol{Y}_{23}$ & $-i \boldsymbol{Y}_{24}$ \\
\hline$\rho_{4}$ & $-i \dot{\boldsymbol{L}}$ & $i \boldsymbol{Y}_{12}$ & $-i\left(\boldsymbol{Y}_{24}+\boldsymbol{X}_{12}-\boldsymbol{X}_{34}\right)$ & $i \boldsymbol{Y}_{14}$ \\
\hline $\boldsymbol{X}_{12}$ & $\dot{\zeta}_{2}$ & $-\dot{\zeta}_{1}$ & $-\boldsymbol{\beta}_{3}+\dot{\boldsymbol{\zeta}}_{4}$ & $-\boldsymbol{\beta}_{4}-\dot{\boldsymbol{\zeta}}_{3}$ \\
\hline $\boldsymbol{X}_{14}$ & $\dot{\zeta}_{4}$ & $\boldsymbol{\beta}_{4}+\dot{\boldsymbol{\zeta}}_{3}$ & $-\boldsymbol{\beta}_{1}-\dot{\boldsymbol{\zeta}}_{2}$ & $-\zeta_{1}$ \\
\hline $\boldsymbol{X}_{23}$ & $-\boldsymbol{\beta}_{3}+\dot{\boldsymbol{\zeta}}_{4}$ & $\dot{\zeta}_{3}$ & $-\dot{\zeta}_{2}$ & $\boldsymbol{\beta}_{2}-\dot{\boldsymbol{\zeta}}_{1}$ \\
\hline $\boldsymbol{X}_{24}$ & $-\boldsymbol{\beta}_{4}-\dot{\boldsymbol{\zeta}}_{3}$ & $\dot{\zeta}_{4}$ & $-\boldsymbol{\beta}_{2}+\dot{\boldsymbol{\zeta}}_{1}$ & $-\dot{\zeta}_{2}$ \\
\hline $\boldsymbol{X}_{34}$ & $\boldsymbol{\beta}_{1}+\dot{\boldsymbol{\zeta}}_{2}$ & $\boldsymbol{\beta}_{2}-\dot{\boldsymbol{\zeta}}_{1}$ & $\dot{\zeta}_{4}$ & $-\dot{\zeta}_{3}$ \\
\hline$Y_{12}$ & $\dot{\rho}_{3}$ & $\dot{\rho}_{4}$ & $-\boldsymbol{\beta}_{2}+\dot{\boldsymbol{\rho}}_{1}$ & $\boldsymbol{\beta}_{1}+\dot{\boldsymbol{\rho}}_{2}$ \\
\hline$Y_{14}$ & $\dot{\rho}_{1}$ & $-\boldsymbol{\beta}_{1}-\dot{\boldsymbol{\rho}}_{2}$ & $-\boldsymbol{\beta}_{4}-\dot{\boldsymbol{\rho}}_{3}$ & $\dot{\boldsymbol{\rho}}_{4}$ \\
\hline $\boldsymbol{Y}_{23}$ & $-\boldsymbol{\beta}_{2}+\dot{\boldsymbol{\rho}}_{1}$ & $-\dot{\boldsymbol{\rho}}_{2}$ & $-\dot{\boldsymbol{\rho}}_{3}$ & $-\boldsymbol{\beta}_{3}+\dot{\boldsymbol{\rho}}_{4}$ \\
\hline $\boldsymbol{Y}_{24}$ & $\boldsymbol{\beta}_{1}+\dot{\boldsymbol{\rho}}_{2}$ & $\dot{\boldsymbol{\rho}}_{1}$ & $\boldsymbol{\beta}_{3}-\dot{\boldsymbol{\rho}}_{4}$ & $-\dot{\boldsymbol{\rho}}_{3}$ \\
\hline $\boldsymbol{Y}_{34}$ & $\boldsymbol{\beta}_{4}+\dot{\boldsymbol{\rho}}_{3}$ & $-\boldsymbol{\beta}_{3}+\dot{\boldsymbol{\rho}}_{4}$ & $\dot{\boldsymbol{\rho}}_{1}$ & $\dot{\rho}_{2}$ \\
\hline $\boldsymbol{\beta}_{1}$ & $i\left(\dot{\boldsymbol{X}}_{34}-\dot{\boldsymbol{X}}_{12}\right)$ & $-i\left(\dot{\boldsymbol{Y}}_{14}-\dot{\boldsymbol{Y}}_{23}\right)$ & $-i\left(\dot{\boldsymbol{X}}_{14}-\dot{\boldsymbol{X}}_{23}\right)$ & $-i\left(\dot{\boldsymbol{Y}}_{34}-\dot{\boldsymbol{Y}}_{12}\right)$ \\
\hline $\boldsymbol{\beta}_{2}$ & $i\left(\dot{\boldsymbol{Y}}_{14}-\dot{\boldsymbol{Y}}_{23}\right)$ & $i\left(\dot{\boldsymbol{X}}_{34}-\dot{\boldsymbol{X}}_{12}\right)$ & $i\left(\dot{\boldsymbol{Y}}_{34}-\dot{\boldsymbol{Y}}_{12}\right)$ & $-i\left(\dot{\boldsymbol{X}}_{14}-\dot{\boldsymbol{X}}_{23}\right)$ \\
\hline $\boldsymbol{\beta}_{3}$ & $i\left(\dot{\boldsymbol{X}}_{14}-\dot{\boldsymbol{X}}_{23}\right)$ & $-i\left(\dot{\boldsymbol{Y}}_{34}-\dot{\boldsymbol{Y}}_{12}\right)$ & $i\left(\dot{\boldsymbol{X}}_{34}-\dot{\boldsymbol{X}}_{12}\right)$ & $i\left(\dot{\boldsymbol{Y}}_{14}-\dot{\boldsymbol{Y}}_{23}\right)$ \\
\hline $\boldsymbol{\beta}_{4}$ & $i\left(\dot{\boldsymbol{Y}}_{34}-\dot{\boldsymbol{Y}}_{12}\right)$ & $\left(\dot{\boldsymbol{X}}_{14}-\dot{\boldsymbol{X}}_{23}\right)$ & $-i\left(\dot{\boldsymbol{Y}}_{14}-\dot{\boldsymbol{Y}}_{23}\right)$ & $i\left(\dot{\boldsymbol{X}}_{34}-\dot{\boldsymbol{X}}_{12}\right)$ \\
\hline
\end{tabular}

Higher-Level Bosons: The transformation results of $\mathrm{D}_{I} \boldsymbol{\zeta}_{J}$ and $\mathrm{D}_{I} \boldsymbol{\rho}_{J}$ involve $6+6$ distinct linear combinations of the $5+5$ independent bosons $\boldsymbol{X}_{I J}$ and $\boldsymbol{Y}_{I J}$. It is therefore impossible to rewrite each of these as a single, linearly independent component field or its derivative, and the CLS supermultiplet is therefore unavoidably non-adinkraic.

We thus turn to the ten higher-level bosons, $\boldsymbol{X}_{I J}, \boldsymbol{Y}_{I J}$, with $I J \neq 13$, and notice that the particular combinations $\boldsymbol{X}_{34}-\boldsymbol{X}_{12}$ and $\boldsymbol{Y}_{34}-\boldsymbol{Y}_{12}$ occur (1) throughout the supersymmetry transformations of $\boldsymbol{\beta}_{I}$, as well as (2) in all trinomial terms in the supersymmetry transformations of $\boldsymbol{\zeta}_{1}, \boldsymbol{\zeta}_{3}, \boldsymbol{\rho}_{2}, \boldsymbol{\rho}_{4}$. Therefore, the redefinitions

$$
\boldsymbol{X}_{34} \rightarrow \boldsymbol{X}_{34}:=\left(\boldsymbol{X}_{34}-\boldsymbol{X}_{12}\right) \quad \text { and } \quad \boldsymbol{Y}_{34} \rightarrow \boldsymbol{Y}_{34}:=\left(\boldsymbol{Y}_{34}-\boldsymbol{Y}_{12}\right)
$$

reduce all trinomials in the results of $\mathrm{D}_{I} \boldsymbol{\zeta}_{J}$ and $\mathrm{D}_{I} \boldsymbol{\rho}_{J}$ into binomials, and also reduce some of the binomials in the results of $\mathrm{D}_{I} \boldsymbol{X}_{J K}$ and $\mathrm{D}_{I} \boldsymbol{Y}_{J K}$. Similar simplification are achieved also by the replacing

$$
\boldsymbol{X}_{14} \rightarrow \boldsymbol{X}_{14}:=\left(\boldsymbol{X}_{14}-\boldsymbol{X}_{23}\right) \quad \text { and } \quad \boldsymbol{Y}_{14} \rightarrow \boldsymbol{Y}_{14}:=\left(\boldsymbol{Y}_{14}-\boldsymbol{Y}_{23}\right)
$$


The component field combinations (3.16a) are related to the primary relations (3.3), while the component field combinations $(3.16 \mathrm{~b})$ are related to the secondary relation $(3.15 \mathrm{c})$.

Table 4: The superderivative relations for the dimensionally reduced complex linear superfield, after the field redefinitions (3.16). The fields and colors are organized to expose the antisymmetric $F_{\mu \nu}$-like structure in the transformations.

\begin{tabular}{c|cccc} 
& $\mathrm{D}_{2}$ & $\mathrm{D}_{4}$ & $\mathrm{D}_{3}$ & $\mathrm{D}_{1}$ \\
\hline $\boldsymbol{K}$ & $\boldsymbol{\zeta}_{2}$ & $\boldsymbol{\zeta}_{4}$ & $\boldsymbol{\zeta}_{3}$ & $\boldsymbol{\zeta}_{1}$ \\
$\boldsymbol{L}$ & $\boldsymbol{\rho}_{3}$ & $\boldsymbol{\rho}_{1}$ & $-\boldsymbol{\rho}_{2}$ & $-\boldsymbol{\rho}_{4}$ \\
\hline $\boldsymbol{X}_{24}$ & $\dot{\boldsymbol{\zeta}}_{4}$ & $-\dot{\boldsymbol{\zeta}}_{2}$ & $\dot{\boldsymbol{\zeta}}_{1}-\boldsymbol{\beta}_{2}$ & $-\dot{\boldsymbol{\zeta}}_{3}-\boldsymbol{\beta}_{4}$ \\
$\boldsymbol{X}_{23}$ & $\dot{\boldsymbol{\zeta}}_{3}$ & $-\dot{\boldsymbol{\zeta}}_{1}+\boldsymbol{\beta}_{2}$ & $-\dot{\boldsymbol{\zeta}}_{2}$ & $\dot{\boldsymbol{\zeta}}_{4}-\boldsymbol{\beta}_{3}$ \\
$\boldsymbol{X}_{21}$ & $\dot{\boldsymbol{\zeta}}_{1}$ & $\dot{\boldsymbol{\zeta}}_{3}+\boldsymbol{\beta}_{4}$ & $-\dot{\boldsymbol{\zeta}}_{4}+\boldsymbol{\beta}_{3}$ & $-\dot{\boldsymbol{\zeta}}_{2}$ \\
$\boldsymbol{Y}_{24}$ & $\dot{\boldsymbol{\rho}}_{1}$ & $-\dot{\boldsymbol{\rho}}_{3}$ & $-\dot{\boldsymbol{\rho}}_{4}+\boldsymbol{\beta}_{3}$ & $\dot{\boldsymbol{\rho}}_{2}+\boldsymbol{\beta}_{1}$ \\
$\boldsymbol{Y}_{23}$ & $-\dot{\boldsymbol{\rho}}_{2}$ & $\dot{\boldsymbol{\rho}}_{4}-\boldsymbol{\beta}_{3}$ & $-\dot{\boldsymbol{\rho}}_{3}$ & $\dot{\boldsymbol{\rho}}_{1}-\boldsymbol{\beta}_{2}$ \\
$\boldsymbol{Y}_{21}$ & $-\dot{\boldsymbol{\rho}}_{4}$ & $-\dot{\boldsymbol{\rho}}_{2}-\boldsymbol{\beta}_{1}$ & $-\dot{\boldsymbol{\rho}}_{1}+\boldsymbol{\beta}_{2}$ & $-\dot{\boldsymbol{\rho}}_{3}$ \\
\cline { 2 - 5 } $\boldsymbol{X}_{43}$ & $-\boldsymbol{\beta}_{2}$ & $-\boldsymbol{\beta}_{4}$ & $-\boldsymbol{\beta}_{3}$ & $-\boldsymbol{\beta}_{1}$ \\
$\boldsymbol{X}_{14}$ & $\boldsymbol{\beta}_{4}$ & $-\boldsymbol{\beta}_{2}$ & $-\boldsymbol{\beta}_{1}$ & $\boldsymbol{\beta}_{3}$ \\
$\boldsymbol{\Psi}_{43}$ & $\boldsymbol{\beta}_{3}$ & $\boldsymbol{\beta}_{1}$ & $-\boldsymbol{\beta}_{2}$ & $-\boldsymbol{\beta}_{4}$ \\
$\boldsymbol{\Psi}_{41}$ & $\boldsymbol{\beta}_{1}$ & $-\boldsymbol{\beta}_{3}$ & $\boldsymbol{\beta}_{4}$ & $-\boldsymbol{\beta}_{2}$ \\
\hline
\end{tabular}

\begin{tabular}{c|cccc} 
& $\mathrm{D}_{2}$ & $\mathrm{D}_{4}$ & $\mathrm{D}_{3}$ & $\mathrm{D}_{1}$ \\
\hline $\boldsymbol{\zeta}_{2}$ & $i \dot{\boldsymbol{K}}$ & $-i \boldsymbol{X}_{24}$ & $-i \boldsymbol{X}_{23}$ & $-i \boldsymbol{X}_{21}$ \\
$\boldsymbol{\zeta}_{4}$ & $i \boldsymbol{X}_{24}$ & $i \dot{\boldsymbol{K}}$ & $-i \boldsymbol{X}_{21}-i \boldsymbol{X}_{43}$ & $i \boldsymbol{X}_{23}-i \boldsymbol{X}_{41}$ \\
$\boldsymbol{\zeta}_{3}$ & $i \boldsymbol{X}_{23}$ & $i \boldsymbol{X}_{21}+i \boldsymbol{X}_{43}$ & $i \dot{\boldsymbol{K}}$ & $-i \boldsymbol{X}_{24}+i \boldsymbol{\Psi}_{43}$ \\
$\boldsymbol{\zeta}_{1}$ & $i \boldsymbol{X}_{21}$ & $-i \boldsymbol{X}_{23}+i \boldsymbol{X}_{41}$ & $i \boldsymbol{X}_{24}-i \boldsymbol{\Psi}_{43}$ & $i \dot{\boldsymbol{K}}$ \\
$\boldsymbol{\rho}_{3}$ & $i \dot{\boldsymbol{L}}$ & $-i \boldsymbol{Y}_{24}$ & $-i \boldsymbol{Y}_{23}$ & $-i \boldsymbol{Y}_{21}$ \\
$\boldsymbol{\rho}_{1}$ & $i \boldsymbol{Y}_{24}$ & $i \dot{\boldsymbol{L}}$ & $-i \boldsymbol{Y}_{21}-i \boldsymbol{\Psi}_{43}$ & $i \boldsymbol{Y}_{23}-i \boldsymbol{\Psi}_{41}$ \\
$-\boldsymbol{\rho}_{2}$ & $i \boldsymbol{Y}_{23}$ & $i \boldsymbol{Y}_{21}+i \boldsymbol{\Psi}_{43}$ & $i \dot{\boldsymbol{L}}$ & $-i \boldsymbol{Y}_{24}-i \boldsymbol{X}_{43}$ \\
$-\boldsymbol{\rho}_{4}$ & $i \boldsymbol{Y}_{21}$ & $-i \boldsymbol{Y}_{23}+i \boldsymbol{\Psi}_{41}$ & $i \boldsymbol{Y}_{24}+i \boldsymbol{X}_{43}$ & $i \dot{\boldsymbol{L}}$ \\
\hline $\boldsymbol{\beta}_{1}$ & $i \dot{\boldsymbol{Y}}_{41}$ & $i \dot{\boldsymbol{Y}}_{43}$ & $i \dot{\boldsymbol{X}}_{41}$ & $-i \dot{\boldsymbol{X}}_{43}$ \\
$-\boldsymbol{\beta}_{3}$ & $-i \dot{\boldsymbol{\Psi}}_{43}$ & $i \dot{\boldsymbol{Y}}_{41}$ & $i \dot{\boldsymbol{X}}_{43}$ & $i \dot{\boldsymbol{X}}_{41}$ \\
$\boldsymbol{\beta}_{4}$ & $-i \dot{\boldsymbol{X}}_{41}$ & $-i \dot{\boldsymbol{X}}_{43}$ & $i \dot{\boldsymbol{Y}}_{41}$ & $-i \dot{\boldsymbol{\Psi}}_{43}$ \\
$-\boldsymbol{\beta}_{2}$ & $i \dot{\boldsymbol{X}}_{43}$ & $-i \dot{\boldsymbol{X}}_{41}$ & $i \dot{\boldsymbol{Y}}_{43}$ & $i \dot{\boldsymbol{Y}}_{41}$ \\
\hline
\end{tabular}

These replacements turn Table 3 into Table 4, written in terms of the optimal basis (3.17)

$$
\left(\boldsymbol{K}, \boldsymbol{L}\left|\boldsymbol{\zeta}_{I}, \boldsymbol{\rho}_{J}\right| \boldsymbol{X}_{21}, \boldsymbol{X}_{23}, \boldsymbol{X}_{24}, \boldsymbol{Y}_{21}, \boldsymbol{Y}_{23}, \boldsymbol{Y}_{24}, \boldsymbol{X}_{41}, \boldsymbol{X}_{43}, \boldsymbol{Y}_{41}, \boldsymbol{Y}_{43} \mid \boldsymbol{\beta}_{I}\right)
$$

The structure shown in Table 4 may be depicted akin to the Adinkras of Refs. [2,4,5,6], provided we introduce an additional graphical element: uni-directional edges, to represent the fact that, e.g., $\mathrm{D}_{4} \boldsymbol{\zeta}_{3} \supset \boldsymbol{X}_{43}$, but $\mathrm{D}_{4} \boldsymbol{X}_{43} \not \supset \dot{\boldsymbol{\zeta}}_{3}$. (Nevertheless, as the results in Table 4 easily show, the algebra (1.2) is satisfied by the $Q_{I}$-action upon each and every component field.) These uni-directional (partial) transformations were depicted as grey edges in the 'blueprint' of Fig. 1. Uni-directional edges were also introduced in Ref. [31] where they too were depicted as grey edges. Herein, to avoid complicating the graphs using four additional colors and to visually indicate their (exclusively) upward direction, we depict uni-directional edges by tapering edges; see Figure 2.

Top-Level Fermions: Further row-operations in the same vein as those used for the higher-level bosons such as $\boldsymbol{\beta}_{1} \rightarrow\left(\boldsymbol{\beta}_{1}+\dot{\boldsymbol{\rho}}_{2}\right)$ so as to remove the non-Adinkraicity from the transformations of the $\boldsymbol{X}_{I J}, \boldsymbol{Y}_{I J}$ bosons would re-introduce second derivatives in the supersymmetry transformation rule:

$$
\mathrm{D}_{3}\left(\boldsymbol{\beta}_{1}+\dot{\boldsymbol{\rho}}_{2}\right)=-i \dot{\boldsymbol{X}}_{14}-i \ddot{\boldsymbol{L}}
$$

In turn, the lower-level fermions, $\boldsymbol{\zeta}_{I}, \boldsymbol{\rho}_{I}$ cannot be redefined by an admixture of the top-level fermions locally. Furthermore, no row-operation amongst the top-level fermions offers any cancellation or qualitative simplification. This leaves $\boldsymbol{\beta}_{I}$ as the optimal choice. 


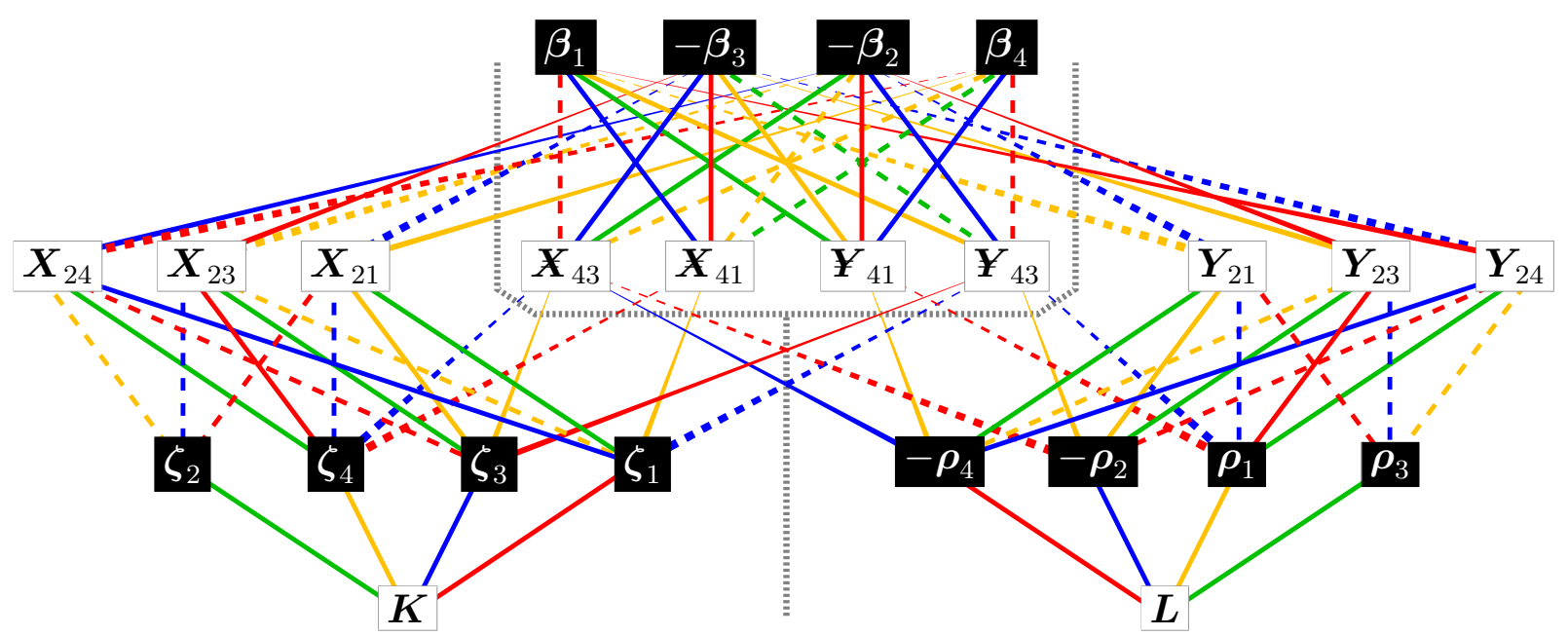

Figure 2: A quasi-Adinkraic graphical depiction of the CLS.

Reduction: Table 4 and Figure 2 make it clear that the top-most component fields,

$$
C L S_{V}:=\left(\boldsymbol{X}_{43}, \boldsymbol{X}_{41}, \boldsymbol{Y}_{41}, \boldsymbol{Y}_{43} \mid \boldsymbol{\beta}_{I}\right)
$$

all by themselves properly close under supersymmetry, and so span a sub-representation, within CLS. Furthermore, the sub-supermultiplet (3.19) is adinkraic and has the chromotopography ${ }^{3}$ of a trans-valise [17]. That is to say, it is possible (in principle) to consistently gauge away the remaining lower components, $\left(\boldsymbol{K}, \boldsymbol{L}\left|\boldsymbol{\zeta}_{I}, \boldsymbol{\rho}_{I}\right| \boldsymbol{X}_{2 J}, \boldsymbol{Y}_{2 J}\right)$, leaving behind the supermultiplet (3.19) as an analogue of the Wess-Zumino gauge-fixed "vector" supermultiplet [14,50]. Thereby, the CLS is reduced, i.e., shown to contain smaller supermultiplets, although it did not decompose into their direct sum.

Now, because of:

1. the previous conclusion that the top-level fermions, $\boldsymbol{\beta}_{I}$ must not be mixed with (the $\partial_{\tau^{-}}$ derivatives of) the lower-level fermions, and

2. the fact that no row-operation in the rows with the $Q$-transformations of the component fields $\boldsymbol{X}_{21}, \boldsymbol{X}_{23}, \boldsymbol{X}_{24}, \boldsymbol{Y}_{21}, \boldsymbol{Y}_{23}, \boldsymbol{Y}_{24}$ can eliminate the remaining binomials,

we conclude that the "other" component fields $\left(\boldsymbol{K}, \boldsymbol{L}\left|\boldsymbol{\zeta}_{I}, \boldsymbol{\rho}_{I}\right| \boldsymbol{X}_{2 J}, \boldsymbol{Y}_{2 J}\right)$ do not form a closed, separate supermultiplet - unless we set

$$
\left(\boldsymbol{X}_{41}, \boldsymbol{X}_{43}, \boldsymbol{Y}_{41}, \boldsymbol{Y}_{43} \mid \boldsymbol{\beta}_{I}\right) \stackrel{!}{=}(0,0,0,0 \mid 0,0,0,0)
$$

whereupon the remainder of $C L S,\left.C L S\right|_{C L S_{V}=0}$, clearly decomposes into

$$
\left(\mathbb{T}_{x}:=\left(\boldsymbol{K}\left|\boldsymbol{\zeta}_{I}\right| \boldsymbol{X}_{21}, \boldsymbol{X}_{23}, \boldsymbol{X}_{24}\right)\right) \oplus\left(\mathbb{T}_{y}:=\left(\boldsymbol{L}\left|\boldsymbol{\rho}_{I}\right| \boldsymbol{Y}_{21}, \boldsymbol{Y}_{23}, \boldsymbol{Y}_{24}\right)\right),
$$

which are also adinkraic, and are recognized as the worldline dimensional reduction of real linear supermultiplets, with dimensions $(1|4| 3|0| 0)$ each. Indeed, notice the $F_{\mu \nu}$-like structure of the $\mathrm{D}_{I^{-}}$ transformations of $\boldsymbol{\zeta}_{I}$ and $\boldsymbol{\rho}_{I}$ in Table 4.

\footnotetext{
${ }^{3}$ Extending the definition of chromotopology [4], chromotopography denotes "chromotopology + height," i.e., the chromotopology with the nodes drawn at the height proportional to the engineering dimension of the corresponding component fields.
} 
Schematically, using only the dimensions of these adinkraic supermultiplets,

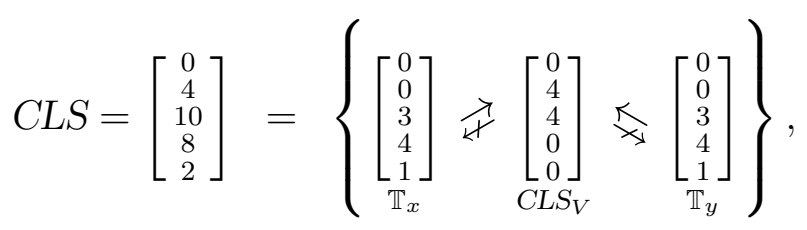

where the arrows indicate that the two "sideline" supermultiplets $\mathbb{T}_{x}$ and $\mathbb{T}_{y} \mathrm{D}$ - $(Q$-) transform into the "central" portion (with higher engineering dimension), CLS $S_{V}$, but not the other way around.

This is reminiscent of the "kites" in Ref. [31, Eq. (7)], but the chromotopographies are rather different: here $\mathbb{T}_{x}$ and $\mathbb{T}_{y}$ are jointly flying the valise kite, $C L S_{V}$. Owing to the "bow-ties theorems" of Ref. [54], it is clear that this valise (3.22) by itself cannot extend even to worldsheet supersymmetry (other than the trivial extension cases of unidextrous (4,0)- and (0,4)-supersymmetry), and therefore certainly does not extend to supersymmetry in any higher-dimensional spacetime. However, the "bow-ties theorems" of Ref. [54] do not apply to gauge-fixed supermultiplets, and it is thus not impossible that $C L S_{V}$ as a gauge-fixed supermultiplet (á la Wess-Zumino) does extend to higher dimensions. In fact, this observation brings us back to the very first presentation [20] of the components of the complex linear multiplet.

In this 'ancient' work in Ref. [20] there appears a discussion highlighting the fact that the fundamental superfield which describes the CLS is actually a gauge spinor superfield. This is true even though none of component fields in the multiplet are gauge fields. Nonetheless the superfield that describes them is a gauge superfield. In fact, the $C L S_{V}$ submultiplet is the analog for the CLS superfield as the Weyl supermultiplet is for the supergravity supermultiplet [19].

Encoding the Reduction: To specify the "sideline" "tensor-like" supermultiplets portions of CLS as proper sub-supermultiplets, we must impose a $Q$-covariant constraint on $C L S$, one that would annihilate the $(0|0| 4|4| 0)$-dimensional "central" portion, $C L S_{V}$, which holds the CLS together. The $Q$-invariance of the superderivatives $\left(\left\{Q_{I}, \mathrm{D}_{J}\right\}=0\right)$ makes the manifestly supersymmetric superfield formalism the natural choice.

Thus, we wish to annihilate the bosons $\boldsymbol{X}_{34}:=\left(\boldsymbol{X}_{34}-\boldsymbol{X}_{12}\right), \boldsymbol{X}_{14}:=\left(\boldsymbol{X}_{14}-\boldsymbol{X}_{23}\right), \boldsymbol{Y}_{34}:=$ $\left(\boldsymbol{Y}_{34}-\boldsymbol{Y}_{12}\right)$, and $\boldsymbol{Y}_{14}:=\left(\boldsymbol{Y}_{14}-\boldsymbol{Y}_{23}\right)$. These combinations are defined as the superderivatives

$$
\begin{array}{ll}
\boldsymbol{X}_{34}=-i \frac{1}{2}\left[\mathrm{D}_{[3} \mathrm{D}_{4]}-\mathrm{D}_{[1} \mathrm{D}_{2]}\right] \boldsymbol{K}, & \boldsymbol{Y}_{34}=-i \frac{1}{2}\left[\mathrm{D}_{[3} \mathrm{D}_{4]}-\mathrm{D}_{[1} \mathrm{D}_{2]}\right] \boldsymbol{L}, \\
\boldsymbol{X}_{14}=-i \frac{1}{2}\left[\mathrm{D}_{[1} \mathrm{D}_{4]}-\mathrm{D}_{[2} \mathrm{D}_{3]}\right] \boldsymbol{K}, & \boldsymbol{Y}_{14}=-i \frac{1}{2}\left[\mathrm{D}_{[1} \mathrm{D}_{4]}-\mathrm{D}_{[2} \mathrm{D}_{3]}\right] \boldsymbol{L} .
\end{array}
$$

This suggests the annihilation of both $\boldsymbol{K}$ and $\boldsymbol{L}$ by the superdifferential operators $\left[\mathrm{D}_{[3} \mathrm{D}_{4]}-\mathrm{D}_{[1} \mathrm{D}_{2]}\right]$ and $\left[\mathrm{D}_{[1} \mathrm{D}_{4]}-\mathrm{D}_{[2} \mathrm{D}_{3]}\right]$ - in addition to the imposition of $(3.1)$.

Indeed, we have the iteration of superdifferential constraints

$$
\left.\mathbb{T}_{x} \oplus \mathbb{T}_{y}=\left\{\left(C^{a b}+\left(\gamma^{5}\right)^{a b}\right) \mathrm{D}_{a} \mathrm{D}_{b}(\boldsymbol{K}+i \boldsymbol{L})=0\right\} \& \begin{array}{l}
{\left[\mathrm{D}_{[3} \mathrm{D}_{4]}-\mathrm{D}_{[1} \mathrm{D}_{2]}\right](\boldsymbol{K}+i \boldsymbol{L})=0} \\
{\left[\mathrm{D}_{[1} \mathrm{D}_{4]}-\mathrm{D}_{[2} \mathrm{D}_{3]}\right](\boldsymbol{K}+i \boldsymbol{L})=0}
\end{array}\right\}
$$

Since $\left[\mathrm{D}_{[3} \mathrm{D}_{4]}-\mathrm{D}_{[1} \mathrm{D}_{2]}\right]$ and $\left[\mathrm{D}_{[1} \mathrm{D}_{4]}-\mathrm{D}_{[2} \mathrm{D}_{3]}\right]$ are real operators, they apply separately on $\boldsymbol{K}$ and $\boldsymbol{L}$, as desired, thus setting all four bosons $\boldsymbol{X}_{34}, \boldsymbol{X}_{14}, \boldsymbol{Y}_{34}$ and $\boldsymbol{Y}_{14}$ to zero. Since the supersymmetry 
transformation of these component fields consists - after the imposition of Eq. (3.7)—purely of $\widetilde{\boldsymbol{\beta}}_{I}$, then supersymmetry implies that the $2^{\text {nd }}$ "tier" of superdifferential constraints (3.24),

$$
\left[\mathrm{D}_{[3} \mathrm{D}_{4]}-\mathrm{D}_{[1} \mathrm{D}_{2]}\right](\boldsymbol{K}+i \boldsymbol{L})=0 \quad \text { and } \quad\left[\mathrm{D}_{[1} \mathrm{D}_{4]}-\mathrm{D}_{[2} \mathrm{D}_{3]}\right](\boldsymbol{K}+i \boldsymbol{L})=0
$$

also annihilates $\widetilde{\boldsymbol{\beta}}_{I}$.

In fact, the superdifferential operators $\left[\mathrm{D}_{[3} \mathrm{D}_{4]}-\mathrm{D}_{[1} \mathrm{D}_{2]}\right]$ and $\left[\mathrm{D}_{[1} \mathrm{D}_{4]}-\mathrm{D}_{[2} \mathrm{D}_{3]}\right]$ appear, at least in the 0-brane reduction, in the constraints that define the $C L S$ itself. The operator $\left[\mathrm{D}_{[3} \mathrm{D}_{4]}-\mathrm{D}_{[1} \mathrm{D}_{2]}\right]$ appears in the primary superdifferential constraint (3.2). The 0-brane reductions, Eq. (3.26),

$$
\begin{aligned}
& H\left[\mathrm{D}_{[1} \mathrm{D}_{4]}-\mathrm{D}_{[2} \mathrm{D}_{3]}\right] \boldsymbol{K}=-2\left[H^{2}+\mathrm{D}_{1} \mathrm{D}_{2} \mathrm{D}_{3} \mathrm{D}_{4}\right] \boldsymbol{L} \\
& 2\left[H^{2}+\mathrm{D}_{1} \mathrm{D}_{2} \mathrm{D}_{3} \mathrm{D}_{4}\right] \boldsymbol{K}=H\left[\mathrm{D}_{[1} \mathrm{D}_{4]}-\mathrm{D}_{[2} \mathrm{D}_{3]}\right] \boldsymbol{L}
\end{aligned}
$$

of the secondary superdifferential constraints (3.8) contains the superdifferential operator $\left[\mathrm{D}_{[1} \mathrm{D}_{4]}-\right.$ $\left.\mathrm{D}_{[2} \mathrm{D}_{3]}\right]$. However, therein, the $\left[\mathrm{D}_{[3} \mathrm{D}_{4]}-\mathrm{D}_{[1} \mathrm{D}_{2]}\right]$ and $\left[\mathrm{D}_{[1} \mathrm{D}_{4]}-\mathrm{D}_{[2} \mathrm{D}_{3]}\right]$ occur only:

1. on one side of the original CLS superdifferential constraints (3.2), and

2. on one side of the 0-brane reduced secondary CLS superdifferential constraints (3.26).

Therefore, imposing (3.25) in addition to (3.2) sets both sides of the equations (3.2) and (3.26) to zero separately. This makes it obvious that $\mathbb{T}_{x} \oplus \mathbb{T}_{y}$, as defined in (3.24), is a special case of CLS, and therefore a sub-supermultiplet,

$$
\mathbb{T}_{x} \oplus \mathbb{T}_{y} \subset C L S, \quad \text { and then } \quad C L S_{V}=C L S /\left(\mathbb{T}_{x} \oplus \mathbb{T}_{y}\right)
$$

Thus, we conclude:

- The CLS is indecomposable as a (1|4)-supermultiplet, but reduces to the direct sum of two smaller (1|4)-supermultiplets, $\mathbb{T}_{x} \oplus \mathbb{T}_{y} \subset C L S$ upon the imposition of the additional superdifferential constraints (3.25).

- The CLS is not adinkraic and it does have uni-directional supersymmetry action. The latter is arguably correlated with the reducibility discussed above: by annihilating the CLS "kite," both the remainder of CLS decomposes and the uni-directional D- (Q-)action is eliminated. 


\section{Actions, Variations, and Conclusions}

\subsection{Real Scalar Supermultiplet}

The $4 D$ transformation laws for the real scalar supermultiplet (2.1) are an invariance of the action

$$
\mathcal{L}_{R S S}=-\frac{1}{2} \boldsymbol{M}^{2}-\frac{1}{2} \boldsymbol{N}^{2}+\frac{1}{2} \boldsymbol{U}_{\mu} \boldsymbol{U}^{\mu}-\frac{1}{2} \boldsymbol{K} \mathbf{d}+i \frac{1}{2} \boldsymbol{\zeta}_{a} C^{a b} \boldsymbol{\Lambda}_{b}
$$

up to total derivatives. Reduced to the 0-brane, this action is unchanged and we now write it in terms of the $\boldsymbol{X}_{I J}$ defined in Eq. (2.5)

$$
\begin{aligned}
\mathcal{L}_{R S S} & =\frac{1}{4} \epsilon^{I J K L} \boldsymbol{X}_{I J} \boldsymbol{X}_{K L}-\frac{1}{2} \boldsymbol{K} \mathbf{d}+i \frac{1}{2} \boldsymbol{\zeta}_{a} C^{a b} \boldsymbol{\Lambda}_{b} \\
& =\boldsymbol{X}_{12} \boldsymbol{X}_{34}+\boldsymbol{X}_{31} \boldsymbol{X}_{24}+\boldsymbol{X}_{14} \boldsymbol{X}_{23}-\frac{1}{2} \boldsymbol{K} \mathbf{d}+i \frac{1}{2}\left(-\boldsymbol{\zeta}_{1} \boldsymbol{\Lambda}_{2}+\boldsymbol{\zeta}_{2} \boldsymbol{\Lambda}_{1}+\boldsymbol{\zeta}_{3} \boldsymbol{\Lambda}_{4}-\boldsymbol{\zeta}_{4} \boldsymbol{\Lambda}_{3}\right) \\
\epsilon^{1234} & =1 \quad \text { and totally antisymmetric. }
\end{aligned}
$$

The Lagrangian (4.2) is clearly invariant up to total derivatives with respect to the 0-brane transformations in Table 1.

\subsection{Real Pseudoscalar Supermultiplet}

Performing the substitutions (2.3) on the RSS action (4.1) we arrive at the Lagrangian for the real pseudoscalar supermultiplet

$$
\mathcal{L}_{R P S}=-\frac{1}{2} \widetilde{\boldsymbol{M}}^{2}-\frac{1}{2} \widetilde{\boldsymbol{N}}^{2}+\frac{1}{2} \boldsymbol{V}_{\mu} \boldsymbol{V}^{\mu}-\frac{1}{2} \boldsymbol{L} \widetilde{\mathbf{d}}+i \frac{1}{2} \boldsymbol{\rho}_{a} C^{a b} \widetilde{\boldsymbol{\Lambda}}_{b}
$$

which is invariant up to total derivatives with respect to the transformation laws (2.4). This action is obviously unchanged upon 0-brane reduction and here we write it in terms of the $\boldsymbol{Y}_{I J}$ defined in Eq. (2.8)

$$
\begin{aligned}
\mathcal{L}_{R P S} & =\frac{1}{4} \epsilon^{I J K L} \boldsymbol{Y}_{I J} \boldsymbol{Y}_{K L}-\frac{1}{2} \boldsymbol{L} \widetilde{\mathbf{d}}+i \frac{1}{2} \boldsymbol{\rho}_{a} C^{a b} \widetilde{\boldsymbol{\Lambda}}_{b} \\
& =\boldsymbol{Y}_{12} \boldsymbol{Y}_{34}+\boldsymbol{Y}_{31} \boldsymbol{Y}_{24}+\boldsymbol{Y}_{14} \boldsymbol{Y}_{23}-\frac{1}{2} \boldsymbol{K} \mathbf{d}+i \frac{1}{2}\left(-\boldsymbol{\rho}_{1} \widetilde{\boldsymbol{\Lambda}}_{2}+\boldsymbol{\rho}_{2} \widetilde{\boldsymbol{\Lambda}}_{1}+\boldsymbol{\rho}_{3} \widetilde{\boldsymbol{\Lambda}}_{4}-\boldsymbol{\rho}_{4} \widetilde{\boldsymbol{\Lambda}}_{3}\right)
\end{aligned}
$$

which is invariant up to total derivatives with respect to the transformation laws in Table 2.

\subsection{Complex Linear Supermultiplet}

The transformation laws (3.10) are an invariant of the CLS Lagrangian (4.6)

$$
\begin{aligned}
\mathcal{L}_{C L S}= & -\frac{1}{4} \partial_{\mu} \boldsymbol{K} \partial^{\mu} \boldsymbol{K}-\frac{1}{4} \partial_{\mu} \boldsymbol{L} \partial^{\mu} \boldsymbol{L}-\frac{1}{2} \boldsymbol{M}^{2}-\frac{1}{2} \boldsymbol{N}^{2}+ \\
& +\frac{1}{4} \boldsymbol{U}_{\mu} \boldsymbol{U}^{\mu}+\frac{1}{4} \boldsymbol{V}_{\mu} \boldsymbol{V}^{\mu}+\frac{1}{2} \boldsymbol{V}^{\mu} \partial_{\mu} \boldsymbol{K}-\frac{1}{2} \boldsymbol{U}^{\mu} \partial_{\mu} \boldsymbol{L} \\
& +\frac{i}{4}\left(\gamma^{\mu}\right)^{a b} \boldsymbol{\zeta}_{a} \partial_{\mu} \boldsymbol{\zeta}_{b}+\frac{i}{4}\left(\gamma^{\mu}\right)^{a b} \boldsymbol{\rho}_{a} \partial_{\mu} \boldsymbol{\rho}_{b}+\frac{i}{4}\left(\boldsymbol{\rho}_{a}+\boldsymbol{\zeta}_{a}\right) C^{a b} \boldsymbol{\beta}_{b}
\end{aligned}
$$

up to total derivatives. We can remove the cross terms

$$
\boldsymbol{V}^{\mu} \partial_{\mu} \boldsymbol{K} \quad, \quad \boldsymbol{U}^{\mu} \partial_{\mu} \boldsymbol{L} \quad, \quad i \boldsymbol{\zeta}_{a} C^{a b} \boldsymbol{\beta}_{b}
$$

and the extra kinetic term

$$
i\left(\gamma^{\mu}\right)^{a b} \boldsymbol{\rho}_{a} \partial_{\mu} \boldsymbol{\rho}_{b}
$$


after switching to the basis

$$
\begin{aligned}
\hat{\boldsymbol{V}}_{\mu} & \equiv \boldsymbol{V}_{\mu}+\partial_{\mu} \boldsymbol{K} \quad, \quad \hat{\boldsymbol{U}}_{\mu} \equiv \boldsymbol{U}_{\mu}-\partial_{\mu} \boldsymbol{L} \\
2 \hat{\boldsymbol{\rho}}_{a} & \equiv \boldsymbol{\rho}_{a}+\boldsymbol{\zeta}_{a} \quad, \quad 2 \hat{\boldsymbol{\zeta}}_{a} \equiv \boldsymbol{\rho}_{a}-\boldsymbol{\zeta}_{a} \\
\hat{\boldsymbol{\beta}}_{a} & \equiv \frac{1}{2} \boldsymbol{\beta}_{a}+\frac{1}{4}\left(\gamma^{\mu}\right)_{a}^{b} \partial_{\mu}\left(\boldsymbol{\rho}_{b}+\zeta_{b}\right)
\end{aligned}
$$

with which the CLS Lagrangian takes, up to total derivatives, the simpler form in (4.10)

$$
\begin{aligned}
\mathcal{L}_{C L S} & =-\frac{1}{2} \partial_{\mu} \boldsymbol{K} \partial^{\mu} \boldsymbol{K}-\frac{1}{2} \partial_{\mu} \boldsymbol{L} \partial^{\mu} \boldsymbol{L}-\frac{1}{2} \boldsymbol{M}^{2}-\frac{1}{2} \boldsymbol{N}^{2}+\frac{1}{4} \hat{\boldsymbol{U}}_{\mu} \hat{\boldsymbol{U}}^{\mu}+\frac{1}{4} \hat{\boldsymbol{V}}_{\mu} \hat{\boldsymbol{V}}^{\mu} \\
& +\frac{1}{2} i\left(\gamma^{\mu}\right)^{a b} \hat{\boldsymbol{\zeta}}_{a} \partial_{\mu} \hat{\boldsymbol{\zeta}}_{b}+i \hat{\boldsymbol{\rho}}_{a} C^{a b} \hat{\boldsymbol{\beta}}_{b} .
\end{aligned}
$$

The 0-brane reduction of the original CLS Lagrangian (4.6) in the original, un-hatted basis takes the form in Eq. (4.11).

$$
\begin{aligned}
\mathcal{L}_{C L S}= & \frac{1}{4} \dot{\boldsymbol{K}}^{2}+\frac{1}{4} \dot{\boldsymbol{L}}^{2}-\frac{1}{4} \boldsymbol{Y}_{14} \dot{\boldsymbol{K}}+\frac{1}{4} \boldsymbol{X}_{14} \dot{\boldsymbol{L}}+\frac{1}{4}\left(\boldsymbol{X}_{23}^{2}+\boldsymbol{X}_{12}^{2}+\boldsymbol{X}_{24}^{2}+\boldsymbol{Y}_{23}^{2}+\boldsymbol{Y}_{12}^{2}+\boldsymbol{Y}_{24}^{2}\right)+ \\
& +\frac{1}{4}\left(\boldsymbol{X}_{14} \boldsymbol{X}_{23}+\boldsymbol{X}_{34} \boldsymbol{X}_{12}+\boldsymbol{Y}_{14} \boldsymbol{Y}_{23}+\boldsymbol{Y}_{34} \boldsymbol{Y}_{23}+\boldsymbol{Y}_{34} \boldsymbol{Y}_{12}+\boldsymbol{Y}_{34} \boldsymbol{X}_{24}-\boldsymbol{X}_{34} \boldsymbol{Y}_{24}\right)+ \\
& +\frac{i}{4} \delta^{I J}\left(\dot{\boldsymbol{\zeta}}_{I} \dot{\boldsymbol{\zeta}}_{J}+\boldsymbol{\rho}_{I} \dot{\boldsymbol{\rho}}_{J}\right)+\frac{i}{4}\left(\boldsymbol{\rho}_{[2} \boldsymbol{\beta}_{1]}+\boldsymbol{\zeta}_{[2} \boldsymbol{\beta}_{1]}+\boldsymbol{\rho}_{[3} \boldsymbol{\beta}_{4]}+\boldsymbol{\zeta}_{[3} \boldsymbol{\beta}_{4]}\right)
\end{aligned}
$$

The field redefinitions (4.9) turn the transformation laws (3.10) into the transformation laws (4.12) for the hatted basis

$$
\begin{aligned}
\mathrm{D}_{a} \boldsymbol{K}= & \hat{\boldsymbol{\rho}}_{a}-\hat{\boldsymbol{\zeta}}_{a} \\
\mathrm{D}_{a} \boldsymbol{L}= & i\left(\gamma^{5}\right)_{a}^{b}\left(\hat{\boldsymbol{\rho}}_{b}+\hat{\boldsymbol{\zeta}}_{b}\right) \\
\mathrm{D}_{a} \hat{\boldsymbol{\rho}}_{b}= & i C_{a b} \boldsymbol{M}+\left(\gamma^{5}\right)_{a b} \boldsymbol{N}+\frac{1}{2}\left(\gamma^{5} \gamma^{\mu}\right)_{a b} \hat{\boldsymbol{U}}_{\mu}+\frac{i}{2}\left(\gamma^{\mu}\right)_{a b} \hat{\boldsymbol{V}}_{\mu} \\
\mathrm{D}_{a} \hat{\boldsymbol{\zeta}}_{b}= & -i\left(\gamma^{\mu}\right)_{a b}\left(\partial_{\mu} \boldsymbol{K}\right)-\left(\gamma^{5} \gamma^{\mu}\right)_{a b}\left(\partial_{\mu} \boldsymbol{L}\right)-\frac{1}{2}\left(\gamma^{5} \gamma^{\mu}\right)_{a b} \hat{\boldsymbol{U}}_{\mu}+\frac{i}{2}\left(\gamma^{\mu}\right)_{a b} \hat{\boldsymbol{V}}_{\mu} \\
\mathrm{D}_{a} \boldsymbol{M}= & \hat{\boldsymbol{\beta}}_{a}-\frac{1}{2}\left(\gamma^{\mu}\right)_{a}{ }^{b}\left(\partial_{\mu} \hat{\boldsymbol{\rho}}_{b}\right) \\
\mathrm{D}_{a} \boldsymbol{N}= & -i\left(\gamma^{5}\right)_{a}{ }^{b} \hat{\boldsymbol{\beta}}_{b}+\frac{i}{2}\left(\gamma^{5} \gamma^{\mu}\right)_{a}^{b}\left(\partial_{\mu} \hat{\boldsymbol{\rho}}_{b}\right) \\
\mathrm{D}_{a} \hat{\boldsymbol{U}}_{\mu}= & i\left(\gamma^{5} \gamma_{\mu}\right)_{a}{ }^{b} \hat{\boldsymbol{\beta}}_{b}-i\left(\gamma^{5}\right)_{a}{ }^{b}\left(\partial_{\mu}\left(\hat{\boldsymbol{\rho}}_{b}+2 \hat{\boldsymbol{\zeta}}_{b}\right)\right)-\frac{i}{2}\left(\gamma^{5} \gamma^{\nu} \gamma_{\mu}\right)_{a}{ }^{b}\left(\partial_{\nu}\left(\hat{\boldsymbol{\rho}}_{b}-2 \hat{\boldsymbol{\zeta}}_{b}\right)\right) \\
\mathrm{D}_{a} \hat{\boldsymbol{V}}_{\mu}= & -\left(\gamma_{\mu}\right)_{a}{ }^{b} \hat{\boldsymbol{\beta}}_{b}+\left(\partial_{\mu}\left(\hat{\boldsymbol{\rho}}_{a}-2 \hat{\boldsymbol{\zeta}}_{a}\right)\right)+\frac{1}{2}\left(\gamma^{\nu} \gamma_{\mu}\right)_{a}{ }^{b}\left(\partial_{\nu}\left(\hat{\boldsymbol{\rho}}_{b}+2 \hat{\boldsymbol{\zeta}}_{b}\right)\right) \\
\mathrm{D}_{a} \hat{\boldsymbol{\beta}}_{b}= & \frac{i}{2}\left(\gamma^{\mu}\right)_{a b}\left(\partial_{\mu} \boldsymbol{M}\right)+\frac{1}{2}\left(\gamma^{5} \gamma^{\mu}\right)_{a b}\left(\partial_{\mu} \boldsymbol{N}\right) \\
& +\frac{1}{2}\left(\gamma^{5} \gamma^{\mu} \gamma^{\nu}\right)_{a b}\left(\partial_{\mu} \hat{\boldsymbol{U}}_{\nu}\right)+\frac{1}{4}\left(\gamma^{5} \gamma^{\nu} \gamma^{\mu}\right)_{a b}\left(\partial_{\mu} \hat{\boldsymbol{U}}_{\nu}\right) \\
& +\frac{i}{2}\left(\gamma^{\mu} \gamma^{\nu}\right)_{a b}\left(\partial_{\mu} \hat{\boldsymbol{V}}_{\nu}\right)+\frac{i}{4}\left(\gamma^{\nu} \gamma^{\mu}\right)_{a b}\left(\partial_{\mu} \hat{\boldsymbol{V}}_{\nu}\right) \\
& +\eta^{\mu \nu} \partial_{\mu} \partial_{\nu}\left(-i C_{a b} \boldsymbol{K}+\left(\gamma^{5}\right)_{a b} \boldsymbol{L}\right)
\end{aligned}
$$

which are an invariant of the $4 D$ CLS Lagrangian (4.10), up to total derivatives. In this hatted basis, the 0-brane reduced Lagrangian takes the most simple form in Eq. (4.13)

$$
\begin{aligned}
\mathcal{L}_{C L S}= & \frac{1}{2} \dot{\boldsymbol{K}}^{2}+\frac{1}{2} \dot{\boldsymbol{L}}^{2}-\frac{1}{2} \boldsymbol{M}^{2}-\frac{1}{2} \boldsymbol{N}^{2}+\frac{1}{4} \hat{\boldsymbol{U}}_{\mu} \hat{\boldsymbol{U}}^{\mu}+\frac{1}{4} \hat{\boldsymbol{V}}_{\mu} \hat{\boldsymbol{V}}^{\mu}+\frac{i}{2} \delta^{I J} \hat{\boldsymbol{\zeta}}_{I} \dot{\hat{\boldsymbol{\zeta}}}_{J}+i\left(\hat{\boldsymbol{\rho}}_{[2} \hat{\boldsymbol{\beta}}_{1]}+\hat{\boldsymbol{\rho}}_{[3} \hat{\boldsymbol{\beta}}_{4]}\right) \\
= & \frac{1}{4} \dot{\boldsymbol{K}}^{2}+\frac{1}{4} \dot{\boldsymbol{L}}^{2}-\frac{1}{4} \boldsymbol{Y}_{14} \dot{\boldsymbol{K}}+\frac{1}{4} \boldsymbol{X}_{14} \dot{\boldsymbol{L}}+\frac{1}{4}\left(\boldsymbol{X}_{23}^{2}+\boldsymbol{X}_{12}^{2}+\boldsymbol{X}_{24}^{2}+\boldsymbol{Y}_{23}^{2}+\boldsymbol{Y}_{12}^{2}+\boldsymbol{Y}_{24}^{2}\right)+ \\
& +\frac{1}{4}\left(\boldsymbol{X}_{14} \boldsymbol{X}_{23}+\boldsymbol{X}_{34} \boldsymbol{X}_{12}+\boldsymbol{Y}_{14} \boldsymbol{Y}_{23}+\boldsymbol{Y}_{34} \boldsymbol{Y}_{23}+\boldsymbol{Y}_{34} \boldsymbol{Y}_{12}+\boldsymbol{Y}_{34} \boldsymbol{X}_{24}-\boldsymbol{X}_{34} \boldsymbol{Y}_{24}\right)+ \\
& +\frac{i}{2} \delta^{I J} \hat{\boldsymbol{\zeta}}_{I} \dot{\hat{\boldsymbol{\zeta}}}_{J}+i\left(\hat{\boldsymbol{\rho}}_{[2} \hat{\boldsymbol{\beta}}_{1]}+\hat{\boldsymbol{\rho}}_{[3} \hat{\boldsymbol{\beta}}_{4]}\right)
\end{aligned}
$$




\subsection{Conclusions}

In this work, we have concentrated on the structure of Adinkra graphs that are inherent in the complex linear superfield $C L S$. Often an overlooked representation in the study of $4 D, \mathcal{N}=1$ supersymmetry, its existence has mostly been viewed as a curiosity that warranted little notice. We have performed a complete analysis of this multiplet in a self-contained explanation of how it is obtained by imposing appropriate super-differential equations on a real scalar and real pseudoscalar superfield in the $4 D, \mathcal{N}=1$ supersymmetry. We have shown that under a '0-brane reduction,' this supermultiplet produces a quasi-Adinkra which demonstrates non-Adinkraicity with respect the $N$-cubical or projected $N$-cubical Adinkras studied previously by the Doran et al. collaboration of Refs. $[2,3,4,5,6]$.

An important implication of this work is that a large class of representations of supersymmetry is provided by Adinkra-like diagrams that are not $N$-cubical. In fact, the construction given in section two of this work (beginning with two $N$-cubical Adinkras for the real scalar and real pseudoscalar supermultiplets) which imposes constraints leads to the non- $N$-cubical Adinkra of the complex linear supermultiplet. In fact, rather than being exceptional, it is more likely that as more complicated supersymmetric systems are considered, the occurrence of non- $N$-cubical Adinkras will become generic. This has implications for the construction of higher dimensional off-shell supermultiplets. Any study based solely on $N$-cubical Adinkras is likely to miss the important class of non-cubical representations. At a minimum such a study would be incomplete and inconclusive.

\section{"A fear of the unknown keeps a lot of people from leaving bad situations." - Kathy Lee Gifford}

Acknowledgments: We thank Sergei M. Kuzenko, Ulf Lindström, Martin Roček, and Warren Siegel for discussions. SJG's and KS's research was supported in part by the endowment of the John S. Toll Professorship, the University of Maryland Center for String \& Particle Theory, National Science Foundation Grant PHY-0354401. SJG's work is also supported by U.S. Department of Energy (D.O.E.) under cooperative agreement DE-FG02-5ER-41360. SJG and KS offer additional gratitude to the M. L. K. Visiting Professorship and to the M. I. T. Center for Theoretical Physics for support and hospitality extended during the undertaking of this work. TH is grateful to the Department of Energy for the generous support through the grant DE-FG02-94ER-40854, as well as the Physics Department of the Faculty of Natural Sciences of the University of Novi Sad, Serbia, for recurring hospitality and resources. 


\section{References}

[1] M. Faux and S. J. Gates, Jr., Adinkras: A graphical technology for supersymmetric representation theory, Phys. Rev. D (3) 71 (2005) 065002.

http://arxiv.org/abs/hep-th/0408004

[2] C. F. Doran, M. G. Faux, S. J. Gates, Jr., T. Hübsch, K. M. Iga and G. D. Landweber, On graph-theoretic identifications of Adinkras, supersymmetry representations and superfields, Int. J. Mod. Phys. A22 (2007) 869-930. http://arxiv.org/abs/math-ph/0512016

[3] C. F. Doran, M. G. Faux, S. J. Gates, Jr., T. Hübsch, K. M. Iga and G. D. Landweber, Off-shell supersymmetry and filtered Clifford supermodules.

http://arXiv.org/abs/math-ph/0603012

[4] C. F. Doran, M. G. Faux, S. J. Gates, Jr., T. Hübsch, K. M. Iga, G. D. Landweber and R. L. Miller, Topology types of Adinkras and the corresponding representations of $N$-extended supersymmetry. http://arxiv.org/abs/0806.0050

[5] C. F. Doran, M. G. Faux, S. J. Gates, Jr., T. Hübsch, K. M. Iga, G. D. Landweber and R. L. Miller, Adinkras for clifford algebras, and worldline supermultiplets.

http://arxiv.org/abs/0811.3410

[6] C. F. Doran, M. G. Faux, S. J. Gates, Jr., T. Hübsch, K. M. Iga and G. D. Landweber, A superfield for every dash-chromotopology, Int. J. Mod. Phys. A24 (2009) 5681-5695.

http://arxiv.org/abs/0901.4970

[7] A. Pashnev and F. Toppan, On the classification of $N$-extended supersymmetric quantum mechanical systems, J. Math. Phys. 42 (2001) 5257-5271.

http://arxiv.org/abs/hep-th/0010135

[8] F. Toppan, Classifying N-extended 1-dimensional supersymmetric systems, in: S. Duplij and J. Wess (Eds.), Noncommutative Structures in Mathematics and Physics, Kluwer Ac. Pub, 2001, p. 195. http://arxiv.org/abs/hep-th/0109047

[9] F. Toppan, Division algebras, extended supersymmetries and applications, Nucl. Phys. Proc. Suppl. 102 (2001) 270-277. http://arxiv.org/abs/hep-th/0109073

[10] H. L. Carrion, M. Rojas and F. Toppan, Octonionic realizations of 1-dimensional extended supersymmetries. a classification, Mod. Phys. Lett. A18 (2003) 787-798.

http://arxiv.org/abs/hep-th/0212030

[11] Z. Kuznetsova, M. Rojas and F. Toppan, Classification of irreps and invariants of the $N$-extended supersymmetric quantum mechanics, JHEP 03 (2006) 098.

http://arxiv.org/abs/hep-th/0511274

[12] Z. Kuznetsova and F. Toppan, Refining the classification of the irreps of the $1 d$ - extended supersymmetry, Mod. Phys. Lett. A23 (2008) 37-51.

http: //arxiv.org/abs/hep-th/0701225 
[13] M. Gonzales, S. Khodaee and F. Toppan, On non-minimal $N=4$ supermultiplets in $1 D$ and their associated sigma-models. http://arXiv.org/pdf/1006.4678

[14] S. J. Gates, Jr., M. T. Grisaru, M. Roček and W. Siegel, Superspace, Benjamin/Cummings Pub. Co., Reading, MA, 1983.

[15] T. Hübsch, Haploid (2,2)-superfields in 2-dimensional space-time, Nucl. Phys. B555(3) (1999) $567-628$.

[16] M. G. Faux, S. J. Gates, Jr. and T. Hübsch, Effective symmetries of the minimal supermultiplet of $N=8$ extended worldline supersymmetry, J. Phys. A42 (2009) 415206. http://arxiv.org/abs/0904.4719

[17] S. J. Gates, Jr., J. Gonzales, B. MacGregor, J. Parker, R. Polo-Sherk, V. G. J. Rodgers and L. Wassink, $4 D, \mathcal{N}=1$ supersymmetry genomics (I), JHEP 12 (2009) 009. http://arXiv.org/abs/0902.3830

[18] S. J. Gates, Jr., J. Hallet, J. Parker, V. G. J. Rodgers and K. Stiffler, $4 D, \mathcal{N}=1$ supersymmetry genomics (II). http://arXiv.org/abs/1112.2147

[19] W. Siegel and S. J. Gates, Jr., Superfield supergravity, Nucl. Phys. B147 (1979) 77-104.

[20] S. J. Gates, Jr. and W. Siegel, Variant Superfield Representations, Nucl. Phys. B187 (1981) 389-396.

[21] B. Zumino, Superspace, in: Erice EPS: Unification, 1980, pp. 101-120.

[22] B. Deo and S. J. Gates, Jr., Comments on nonminimal N=1 scalar multiplets, Nucl. Phys. B254 (1985) 187-200.

[23] S. J. Gates, Jr., Why auxiliary fields matter: The strange case of the $4 D, N=1$ supersymmetric QCD effective action, Phys. Lett. B365 (1996) 132-146. http://arxiv.org/abs/hep-th/9508153

[24] S. J. Gates, Jr., Why auxiliary fields matter: The strange case of the $4 D, N=1$ supersymmetric QCD effective action (II), Nucl. Phys. B485 (1997) 145-189. http://arxiv.org/abs/hep-th/9606109

[25] S. J. Gates, Jr., S. Penati and G. Tartaglino-Mazzucchelli, 6D supersymmetry, projective superspace and 4D, $N=1$ superfields, JHEP 0605 (2006) 051-078. http://arxiv.org/abs/hep-th/0508187

[26] S. J. Gates, Jr., S. Penati and G. Tartaglino-Mazzucchelli, 6D supersymmetric nonlinear sigma-models in 4D, N=1 superspace, JHEP 0609 (2006) 006-061. http://arxiv.org/abs/hep-th/0604042

[27] S. J. Gates, Jr. and S. M. Kuzenko, The CNM-hypermultiplet nexus, Nucl. Phys. B543 (1999) 122-145. http://arxiv.org/abs/hep-th/9810137 
[28] S. J. Gates, Jr., T. Hübsch and S. M. Kuzenko, CNM models, holomorphic functions and projective superspace C-maps, Nucl. Phys. B557 (1999) 443-461.

http://arxiv.org/abs/hep-th/9902211

[29] S. J. Gates, Jr. and S. M. Kuzenko, $4 D N=2$ supersymmetric off-shell sigma models on the cotangent bundles of kähler manifolds, Fortschr. Phys. 48(1-3) (2000) 115-121.

http://arxiv.org/abs/hep-th/9903013

[30] C. F. Doran, M. G. Faux, S. J. Gates, Jr., T. Hübsch, K. M. Iga and G. D. Landweber, Adinkras and the dynamics of superspace prepotentials, Adv. S. Th. Phys. 2(3) (2008)

113-164. http://arXiv.org/abs/hep-th/0605269

[31] C. F. Doran, M. G. Faux, S. J. Gates, Jr., T. Hübsch, K. M. Iga and G. D. Landweber, Frames for supersymmetry, Int. J. Mod. Phys. A 24 (2009) 2665-2676.

http://arxiv.org/abs/0809.5279

[32] S. M. Kuzenko, On compactified harmonic/projective superspace, 5-D superconformal theories, and all that, Nucl. Phys. B745 (2006) 176-207. http://arxiv.org/abs/hep-th/0601177

[33] S. M. Kuzenko, On superpotentials for nonlinear sigma-models with eight supercharges, Phys. Lett. B638 (2006) 288-291. http://arxiv.org/abs/hep-th/0601177

[34] S. M. Kuzenko, U. Lindstrom and R. von Unge, Polar supermultiplets, Hermitian symmetric spaces and hyperkahler metrics, JHEP 0712 (2007) 008-025.

http://arxiv.org/abs/0709.2633

[35] S. M. Kuzenko, On superconformal projective hypermultiplets, JHEP 0712 (2007) 010-040. http://arxiv.org/abs/0710.1479

[36] M. Arai, S. M. Kuzenko and U. Lindstrom, Projective superspace and hyperkahler sigma models on cotangent bundles of Hermitian symmetric spaces, in: AIP Conf. Proc. 957, 2007, pp. 293-296. http://arxiv.org/abs/0709.1257

[37] M. Arai, S. M. Kuzenko and U. Lindstrom, Hyperkahler sigma models on cotangent bundles of Hermitian symmetric spaces using projective superspace, JHEP 0702 (2007) 100-147. http://arxiv.org/abs/0709.1257

[38] S. M. Kuzenko and J. Novak, Chiral formulation for hyperkaehler sigma-models on cotangent bundles of symmetric spaces, JHEP 0812 (2008) 072-083.

http://arxiv.org/abs/0811.0218

[39] S. M. Kuzenko, $N=2$ supersymmetric sigma-models and duality, JHEP 1001 (2010) 115-146. http://arxiv.org/abs/0910.5771

[40] S. M. Kuzenko, U. Lindstrom and R. von Unge, New extended superconformal sigma models and quaternion kähler manifolds, JHEP 0909 (2009) 119-150. 
[41] S. M. Kuzenko, U. Lindstrom and R. von Unge, New supersymmetric sigma-model duality, JHEP 1010 (2010) 072-113.

[42] S. M. Kuzenko, Lectures on nonlinear sigma-models in projective superspace, J. Phys. A43 (2010) 443001-443058. http://arxiv.org/abs/1004.0880

[43] D. Butter and S. M. Kuzenko, $N=2$ supersymmetric sigma-models in AdS, Phys. Lett. B703 (2011) 620-626. http://arxiv.org/abs/1105.3111

[44] D. Butter and S. M. Kuzenko, The structure of $N=2$ supersymmetric nonlinear sigma models in $A d S_{4}$, JHEP 1111 (2011) 080-164. http://arxiv.org/abs/1108.5290

[45] S. M. Kuzenko, Comments on $N=2$ supersymmetric sigma models in projective superspace. http://arxiv.org/abs/1110.4298

[46] D. Butter and S. M. Kuzenko, A dual formulation of supergravity-matter theories, Nucl. Phys. B854 (2012) 1-20. http://arxiv.org/abs/1106.3038

[47] S. M. Kuzenko and S. Tyler, Complex linear superfield as a model for goldstino, JHEP 1104 (2011) 057-066. http://arxiv.org/abs/1102.3042

[48] T. Hübsch, Linear and chiral superfields are usefully inequivalent, Class. Quant. Grav. 16 (1999) L51-154. http://arXiv.org/abs/hep-th/9903175

[49] J. Louis, private communication.

[50] I. L. Buchbinder and S. M. Kuzenko, Ideas and methods of supersymmetry and supergravity, Studies in High Energy Physics Cosmology and Gravitation, IOP Publishing Ltd., Bristol, 1998.

[51] T. Hübsch and G. Katona, On the construction and the structure of off-shell supermultiplet quotients. forthcoming

[52] C. F. Doran,, M.G. Faux, S.J. Gates, Jr., T. Hübsch, K. M. Iga and G. D. Landweber, Adinkras and the Dynamics of Superspace Prepotentials, Adv. S. Th. Phys. 2(3) (2008) 113-164. http://arXiv.org/abs/hep-th/0605269

[53] Gnomon(figure), Wikipedia, The Free Encyclopedia, Wikimedia Foundation, Inc., 24 Jan 2012, Web, 18 Feb 2012. http://en.wikipedia.org/wiki/Gnomon_(figure)

[54] S. J. Gates, Jr. and T. Hübsch, On dimensional extension of supersymmetry: From worldlines to worldsheets. http://arxiv.org/pdf/1104.0722 